\title{
Measurement of the $B^{0}$ Production Cross Section in $p p$ Collisions at $\sqrt{s}=7 \mathrm{TeV}$
}

\author{
S. Chatrchyan et al.* \\ (CMS Collaboration)
}

(Received 14 April 2011; published 20 June 2011)

\begin{abstract}
Measurements of the differential production cross sections $d \sigma / d p_{T}^{B}$ and $d \sigma / d y^{B}$ for $B^{0}$ mesons produced in $p p$ collisions at $\sqrt{s}=7 \mathrm{TeV}$ are presented. The data set used was collected by the CMS experiment at the LHC and corresponds to an integrated luminosity of $40 \mathrm{pb}^{-1}$. The production cross section is measured from $B^{0}$ meson decays reconstructed in the exclusive final state $J / \psi K_{S}^{0}$, with the subsequent decays $J / \psi \rightarrow \mu^{+} \mu^{-}$and $K_{S}^{0} \rightarrow \pi^{+} \pi^{-}$. The total cross section for $p_{T}^{B}>5 \mathrm{GeV}$ and $\left|y^{B}\right|<2.2$ is measured to be $33.2 \pm 2.5 \pm 3.5 \mu \mathrm{b}$, where the first uncertainty is statistical and the second is systematic.
\end{abstract}

DOI: 10.1103/PhysRevLett.106.252001

PACS numbers: $13.85 . \mathrm{Rm}, 12.38 . \mathrm{Bx}, 14.40 . \mathrm{Nd}$

Cross sections for heavy quark production in hard scattering interactions have been studied at $p \bar{p}$ colliders at center-of-mass energies from $630 \mathrm{GeV}$ [1] to $1.96 \mathrm{TeV}$ [2-4] and in $p$-nucleus collisions with beam energies from 800 to $920 \mathrm{GeV}$ [5]. The expected cross sections can be calculated in perturbative quantum chromodynamics. The comparison between data and predictions provides a critical test of next-to-leading order (NLO) calculations [6]. Considerable progress has been achieved in understanding heavy quark production at Tevatron energies, largely resolving earlier discrepancies [7], but substantial theoretical uncertainties remain due to the dependence on the renormalization and factorization scales. Measurements of $b$-hadron production at $7 \mathrm{TeV}$ provided by the Large Hadron Collider (LHC) [8-10] represent a test at a new center-of-mass energy of theoretical approaches that aim to describe heavy flavor production $[11,12]$.

This Letter presents the first measurement of the $B^{0}$ cross section in $p p$ collisions at $\sqrt{s}=7 \mathrm{TeV}$. Events with $B^{0}$ mesons reconstructed from their decays to the final state $J / \psi K_{S}^{0}$, with $J / \psi \rightarrow \mu^{+} \mu^{-}$and $K_{S}^{0} \rightarrow$ $\pi^{+} \pi^{-}$, are used to measure $d \sigma / d p_{T}^{B}, d \sigma / d y^{B}$, and the integrated cross section for transverse momentum $p_{T}^{B}>5 \mathrm{GeV}$ and rapidity $\left|y^{B}\right|<2.2$, where $y$ is defined as $\frac{1}{2} \ln \frac{E+p_{L}}{E-p_{L}}, E$ is the particle energy, and $p_{L}$ is the particle momentum along the counterclockwise beam direction. As the $B^{0}$ and $\bar{B}^{0}$ are indistinguishable in this analysis, both mesons are referred to as $B^{0}$ for the purposes of reconstruction and the final results are divided by two to obtain an average.

The data sample collected by the Compact Muon Solenoid (CMS) detector at the LHC corresponds to an

*Full author list given at the end of the article.

Published by the American Physical Society under the terms of the Creative Commons Attribution 3.0 License. Further distribution of this work must maintain attribution to the author(s) and the published article's title, journal citation, and DOI. integrated luminosity of $39.6 \pm 1.6 \mathrm{pb}^{-1}$ and represents the entire 2010 data set. A detailed description of the detector may be found elsewhere [13]. The main detector components used in this analysis are the silicon tracker and the muon systems.

The silicon tracker measures charged particles within the pseudorapidity range $|\eta|<2.5$, where $\eta=-\ln \tan \left(\frac{\theta}{2}\right)$ and $\theta$ is the polar angle of the track relative to the counterclockwise beam direction. It consists of 1440 silicon pixel and 15148 silicon strip detector modules and is located in the $3.8 \mathrm{~T}$ field of the superconducting solenoid. It provides an impact parameter resolution of $\sim 15 \mu \mathrm{m}$ and a $p_{T}$ resolution of about $1.5 \%$ for particles with transverse momenta up to $100 \mathrm{GeV}$. Muons are measured in the pseudorapidity range $|\eta|<2.4$, with detection planes made using three technologies: drift tubes, cathode strip chambers, and resistive plate chambers.

Events are selected by a trigger requiring two muons without any explicit requirement on the muon momentum. The muon candidates are fully reconstructed offline, combining information from the silicon tracker and muon detectors, and are required to be within the following kinematic acceptance region: $p_{T}^{\mu}>3.3 \mathrm{GeV}$ for $\left|\eta^{\mu}\right|<$ 1.3; total momentum $p^{\mu}>2.9 \mathrm{GeV}$ for $1.3<\left|\eta^{\mu}\right|<2.2$; and $p_{T}^{\mu}>0.8 \mathrm{GeV}$ for $2.2<\left|\eta^{\mu}\right|<2.4$. Opposite-sign muon pairs are fit to a common vertex to form $\mathrm{J} / \psi$ candidates, which are required to be within $150 \mathrm{MeV}$ of the world-average $J / \psi$ mass [14].

The $K_{S}^{0}$ candidates are formed by fitting oppositely charged tracks reconstructed with the CMS tracking algorithm [15] to a common vertex. Each track is required to have at least 6 hits in the silicon tracker, a normalized $\chi^{2}<5$, and a transverse impact parameter with respect to the luminous region greater than 0.5 times its uncertainty. The reconstructed $K_{S}^{0}$ decay vertex must have a normalized $\chi^{2}<7$ and a transverse separation from the luminous region at least 5 times larger than the uncertainty on the separation. The $\pi^{+} \pi^{-}$invariant mass $m_{K_{S}^{0}}$ is required to satisfy $478<m_{K_{S}^{0}}<518 \mathrm{MeV}$, and the reconstructed 
mass distribution is found to be in good agreement with the world-average value [14].

The $B^{0}$ candidates are formed by combining a $J / \psi$ candidate with a $K_{S}^{0}$ candidate. A kinematic fit is performed with the two muons and the $K_{S}^{0}$ candidate, in which the invariant masses of the $J / \psi$ and $K_{S}^{0}$ candidates are constrained to their world-average values [14]. The $B^{0}$ vertex fit confidence level is required to be greater than $1 \%$ and the reconstructed $B^{0}$ mass $m_{B}$ must satisfy $4.9<m_{B}<$ $5.7 \mathrm{GeV}$. When more than one candidate in a single event passes all the selection criteria, only the candidate with the highest $B^{0}$ vertex fit confidence level is retained, which results in the correct choice $99 \%$ of the time in simulated events containing a true signal candidate. A total of 23174 $B^{0}$ candidates pass all selection criteria.

The efficiency of the $B^{0}$ reconstruction is computed with a combination of techniques using the data and large samples of fully simulated signal events generated by PYTHIA 6.422 [16], decayed by EVTGEN [17], and simulated by GEANT4 [18]. The trigger and muon-reconstruction efficiencies are obtained from a large sample of inclusive $J / \psi \rightarrow \mu^{+} \mu^{-}$decays in data using a technique similar to that described in Ref. [19], where one muon is identified with stringent quality requirements, and the second muon is identified using information either exclusively from the tracker (to measure the trigger and muon-identification efficiencies), or from the muon system (to measure the silicon tracking efficiency). Since the dimuon efficiencies are calculated as the product of the measured single muon efficiencies, a correction (1\%-6\%), obtained from the simulation, is applied to take into account efficiency correlations between the two muons. The probabilities for the muons to lie within the kinematic acceptance region and for the $B^{0}$ and $K_{S}^{0}$ candidates to pass the selection requirements are determined from the simulated events. To minimize the effect of the PYTHIA modeling of the $p_{T}^{B}$ and $\left|y^{B}\right|$ distributions on the efficiency calculation, the simulated events are reweighted to match the kinematic distributions observed in the data. The efficiencies for hadron-track reconstruction [20], $K_{S}^{0}$ reconstruction [21], and for fulfilling the vertex quality requirement are found to be consistent between data and simulation within the available precision (up to $5 \%$ ).

The proper decay length of each selected $B^{0}$ candidate is calculated as $c t=\left(m_{B} / p_{T}^{B}\right) L_{x y}$, where the transverse decay length $L_{x y}$ is the vector $\vec{s}$ pointing from the primary vertex [15] to the $B^{0}$ vertex projected onto the $B^{0}$ transverse momentum vector: $L_{x y}=\left(\vec{s} \cdot \vec{p}_{T}^{B}\right) /\left|\vec{p}_{T}^{B}\right|$.

Backgrounds are dominated by prompt and nonprompt $J / \psi$ production, with nonprompt contributions from sources peaking and nonpeaking in $m_{B}$, as shown in Fig. 1. In particular, misreconstructed $b$-hadron decays to final states with a $J / \psi$, such as $B \rightarrow J / \psi K^{*}(892)$, produce a broadly peaking structure in the region $m_{B}<5.2 \mathrm{GeV}$. A study of the dimuon invariant mass distribution confirms
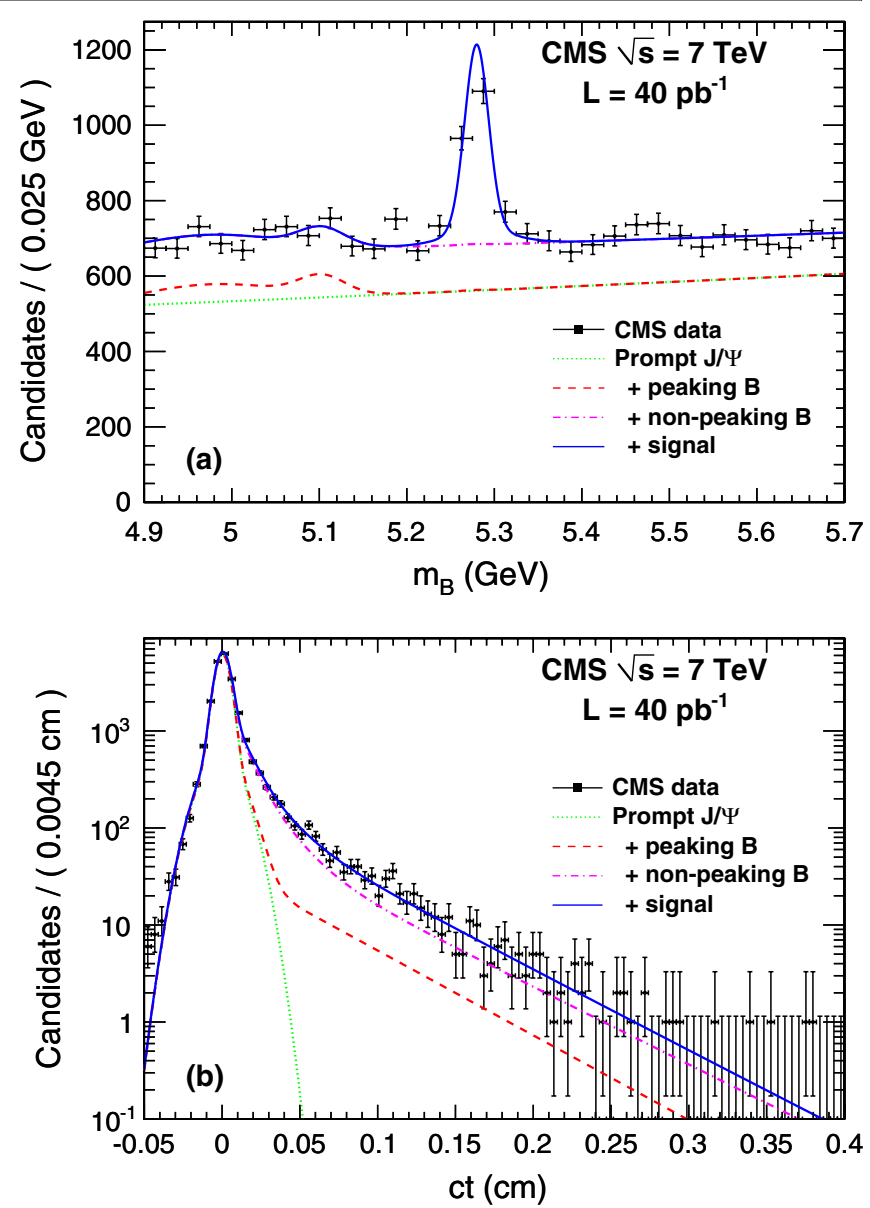

FIG. 1 (color online). Projections of the fit results in (a) $m_{B}$ and (b) $c t$ for $p_{T}^{B}>5 \mathrm{GeV}$ and $\left|y^{B}\right|<2.2$. The curves in each plot are as follows: the sum of all contributions (blue solid line); the prompt $J / \psi$ (green dotted); the sum of the prompt $J / \psi$ and peaking background (red dashed), and the sum of all backgrounds (purple dot-dashed).

that the contamination from events containing a misidentified $J / \psi$ is negligible after all selection criteria have been applied.

The signal yields in each $p_{T}^{B}$ and $\left|y^{B}\right|$ bin are obtained using an unbinned extended maximum-likelihood fit to $m_{B}$ and $c t$. The likelihood for event $j$ is obtained by summing the product of yield $n_{i}$ and probability density $\mathcal{P}_{i}$ for each of the signal and background hypotheses $i$. Four individual components are considered: signal events, prompt $J / \psi$ events, nonprompt $b \rightarrow J / \psi$ events that peak in $m_{B}$ (peaking), and nonprompt $b \rightarrow J / \psi$ events that do not peak in $m_{B}$ (nonpeaking). The extended likelihood function is the product of likelihoods for all events:

$$
\mathcal{L}=\exp \left(-\sum_{i=1}^{4} n_{i}\right) \prod\left[\sum_{j}^{4} n_{i} \mathcal{P}_{i}\left(m_{B} ; \vec{\alpha}_{i}\right) \mathcal{P}_{i}\left(c t ; \vec{\beta}_{i}\right)\right] .
$$

The probability density functions (PDFs), $\mathcal{P}_{i}$, with shape parameters $\vec{\alpha}_{i}$ for $m_{B}$ and $\vec{\beta}_{i}$ for $c t$, are evaluated 
separately for each of the $i$ fit components. The yields $n_{i}$ are determined by maximizing $\mathcal{L}$ with respect to the yields and a subset of the PDF parameters.

The PDF shapes are described below with the parameters obtained from data when possible. The $m_{B}$ PDFs are as follows: the sum of two Gaussian functions for the signal; exponential functions for the prompt and nonpeaking backgrounds; and a sum of three Gaussian functions for the peaking background. The resolution on $m_{B}$ for correctly reconstructed signal events from simulation is approximately $20 \mathrm{MeV}$. The $c t$ PDFs are as follows: a single exponential function convolved with the resolution function to describe the signal and peaking background components, where the lifetimes are allowed to be different; the sum of two exponential functions convolved with the resolution function for the nonpeaking component; and the pure resolution function for the prompt $J / \psi$ component. The resolution function, a sum of two Gaussian functions, is common for signal and background and is measured in data to have an average resolution of $71 \mu \mathrm{m}$.

The fit proceeds in several steps such that all background shapes are obtained directly from data, except for the peaking component which is taken from simulation, as are the signal $m_{B}$ shapes. This technique relies on the assumption that in the region $5.4<m_{B}<5.7 \mathrm{GeV}$ (sideband) there are only two contributions: prompt $J / \psi$ and nonpeaking background. To obtain the effective lifetime distribution of the nonpeaking background, the $m_{B}$ and $c t$ distributions in the $m_{B}$ sideband region are fit simultaneously for events in the inclusive $B^{0}$ sample defined by $p_{T}^{B}>5 \mathrm{GeV}$ and $\left|y^{B}\right|<2.2$. In the second step, the signal $B^{0}$ lifetime in the inclusive sample is determined by fitting $c t$ and $m_{B}$ simultaneously in the full $m_{B}$ range. The result, $c \tau=479 \pm 22 \mu \mathrm{m}$ (statistical uncertainty only), is in agreement with the world-average value, $457 \pm 3 \mu \mathrm{m}$ [14]. With the effective lifetimes for signal and nonprompt background fixed, the signal and background yields are fit in each bin of $p_{T}^{B}$ and $\left|y^{B}\right|$, together with the parameters describing the $c t$ resolution and the shapes of the prompt and nonpeaking components in $m_{B}$.

The accuracy and robustness of the fit strategy were demonstrated by performing a large set of pseudoexperiments, with each one corresponding to the yields observed in data, where signal and background events were generated randomly from the PDFs in each bin. No significant biases were observed on the yields, and the statistical precision of the test was taken as the systematic uncertainty due to potential biases in the fit method. The fit uncertainties were also observed to be estimated properly.

The fitted signal yields in each bin of $p_{T}^{B}$ and $\left|y^{B}\right|$ are summarized in Table I. Figure 1 shows the fit projections for $m_{B}$ and $c t$ from the inclusive sample with $p_{T}^{B}>5 \mathrm{GeV}$ and $\left|y^{B}\right|<2.2$. The total number of signal events is $809 \pm 39$, where the uncertainty is statistical only.

The differential cross section is calculated in bins of $p_{T}^{B}$ as

$$
\frac{d \sigma\left(p p \rightarrow B^{0} X\right)}{d p_{T}^{B}}=\frac{n_{\mathrm{sig}}}{2 \epsilon \mathcal{B} L \Delta p_{T}^{B}}
$$

and similarly for $\left|y^{B}\right|$, where $n_{\text {sig }}$ is the fitted number of signal events in the given bin, $\epsilon$ is the efficiency for a $B^{0}$ meson to pass all the selection criteria, $L$ is the integrated luminosity, $\Delta p_{T}^{B}$ is the bin size, and $\mathcal{B}$ is the product of branching fractions $\mathcal{B}\left(B^{0} \rightarrow J / \psi K_{S}^{0}\right)=(4.36 \pm 0.16) \times$ $10^{-4}, \quad \mathcal{B}\left(\mathrm{J} / \psi \rightarrow \mu^{+} \mu^{-}\right)=(5.93 \pm 0.06) \times 10^{-2}$, and $\mathcal{B}\left(K_{S}^{0} \rightarrow \pi^{+} \pi^{-}\right)=0.6920 \pm 0.0005$ [14]. The additional factor of 2 in the denominator accounts for our choice of quoting the cross section for $B^{0}$ production only, while $n_{\text {sig }}$ includes both $B^{0}$ and $\bar{B}^{0}$. The efficiencies are calculated separately for each bin, always considering only mesons produced with $\left|y^{B}\right|<2.2\left(p_{T}^{B}>5 \mathrm{GeV}\right)$ for $p_{T}^{B}\left(\left|y^{B}\right|\right)$ bins, and take into account bin-to-bin migrations $(<1 \%)$ due to the resolution on the measured $p_{T}^{B}$ and $\left|y^{B}\right|$.

TABLE I. Signal yield $n_{\text {sig }}$, efficiency $\epsilon$, and measured differential cross sections $d \sigma / d p_{T}^{B}$ and $d \sigma / d y^{B}$, compared to the MC@NLO [22] and PYTHIA [16] predictions. The uncertainties in the measured cross sections are statistical and systematic, respectively, excluding the common luminosity (4\%) and branching fraction $(3.8 \%)$ uncertainties. The uncertainties on the signal yields are statistical only, while those on the efficiencies are systematic.

\begin{tabular}{|c|c|c|c|c|c|}
\hline$p_{T}^{B}(\mathrm{GeV})$ & $n_{\text {sig }}$ & $\epsilon(\%)$ & $d \sigma / d p_{T}^{B}(\mu \mathrm{b} / \mathrm{GeV})$ & MC@NLO & PYTHIA \\
\hline $5-10$ & $240 \pm 23$ & $0.65 \pm 0.05$ & $5.20 \pm 0.50 \pm 0.59$ & 3.66 & 7.42 \\
\hline $10-13$ & $169 \pm 17$ & $3.32 \pm 0.28$ & $1.196 \pm 0.121 \pm 0.117$ & 1.13 & 2.14 \\
\hline $13-17$ & $193 \pm 16$ & $6.37 \pm 0.51$ & $0.535 \pm 0.045 \pm 0.051$ & 0.49 & 0.83 \\
\hline $17-24$ & $138 \pm 13$ & $9.60 \pm 0.76$ & $0.145 \pm 0.014 \pm 0.014$ & 0.15 & 0.24 \\
\hline $24-40$ & $70 \pm 9$ & $11.40 \pm 1.04$ & $0.027 \pm 0.003 \pm 0.003$ & 0.025 & 0.035 \\
\hline$\left|y^{B}\right|$ & $n_{\text {sig }}$ & $\epsilon(\%)$ & $d \sigma / d y^{B}(\mu \mathrm{b})$ & MC@NLO & PYTHIA \\
\hline $0.0-0.5$ & $145 \pm 14$ & $1.34 \pm 0.10$ & $7.63 \pm 0.74 \pm 0.76$ & 6.21 & 12.41 \\
\hline $0.5-1.0$ & $141 \pm 15$ & $1.38 \pm 0.10$ & $7.20 \pm 0.75 \pm 0.71$ & 6.14 & 12.01 \\
\hline $1.0-1.4$ & $167 \pm 17$ & $1.93 \pm 0.15$ & $7.61 \pm 0.77 \pm 0.83$ & 5.81 & 11.24 \\
\hline $1.4-1.8$ & $229 \pm 21$ & $2.51 \pm 0.21$ & $8.06 \pm 0.74 \pm 0.89$ & 5.38 & 10.36 \\
\hline $1.8-2.2$ & $128 \pm 17$ & $1.69 \pm 0.14$ & $6.71 \pm 0.87 \pm 0.80$ & 4.81 & 9.26 \\
\hline
\end{tabular}


The cross section is affected by systematic uncertainties on the signal yield and efficiencies, which are uncorrelated bin-to-bin and can affect the shapes of the distributions, and by uncertainties on the branching fractions and luminosity, which are common to all bins and only affect the overall normalization. The uncertainty on the signal yield arises from potential fit biases and imperfect knowledge of the PDF parameters $(4 \%-7 \%)$, and from effects of finalstate radiation and mismeasured track momenta on the signal shape in $m_{B}(1 \%)$. Uncertainties on the efficiencies arise from the trigger $(2 \%-3 \%)$, muon identification $(1 \%)$, muon tracking $(1 \%), K_{S}^{0}(5 \%)$ and $B^{0}(3 \%)$ candidate selection requirements, acceptance (2\%-3\%), dimuon correlations (1\%-5\%) and $p_{T}^{B}$ and $\left|y^{B}\right|$ mismeasurement (1\%). The first five efficiency uncertainties are determined directly from data, while the last three are determined by simulation. The largest uncertainties on the efficiency arise from the $K_{S}^{0}$ reconstruction, which is dominated by the displaced hadronic track efficiency and is measured by comparing the reconstructed $K_{S}^{0}$ lifetime with the known value, and the dimuon correlation uncertainty, which is taken as $100 \%$ of the correction applied to account for the correlations. The difference between the kinematically reweighted and unreweighted results $(3 \%-5 \%)$ is taken as an additional systematic uncertainty. The bin-to-bin systematic uncertainty is computed as the sum in quadrature of the individual uncertainties, and is summarized in Table I. In addition, there are normalization uncertainties of $4 \%$ from the luminosity measurement and of $3.8 \%$ from the branching fractions [14].

The differential cross sections as functions of $p_{T}^{B}$ and $\left|y^{B}\right|$ are shown in Fig. 2 and Table I. They are compared to the predictions of MC@NLO [22] using a $b$-quark mass $m_{b}$ of $4.75 \mathrm{GeV}$, renormalization and factorization scales $\mu=\sqrt{m_{b}^{2}+p_{T}^{2}}$, and the CTEQ6M parton distribution functions [23]. The uncertainty on the predicted cross section is calculated by independently varying the renormalization and factorization scales by factors of two, $m_{b}$ by $\pm 0.25 \mathrm{GeV}$, and by using the CTEQ6.6 parton distribution functions. For reference, the prediction of PYTHIA [16] is also included, using a $b$-quark mass of $4.80 \mathrm{GeV}$, CTEQ6L1 parton distribution functions [23], and the $\mathrm{Z} 2$ tune [24] to simulate the underlying event. The measured $p_{T}$ spectrum falls slightly faster than predicted by MC@NLO, while the $y$ spectrum is measured to be flatter than the PYTHIA prediction and in agreement with the MC@NLO prediction within uncertainties. The integrated cross section for $p_{T}^{B}>5 \mathrm{GeV}$ and $\left|y^{B}\right|<2.2$ is calculated as the sum over all $p_{T}$ bins, without an upper limit for the highest $p_{T}$ bin, to be $33.2 \pm 2.5 \pm 3.5 \mu \mathrm{b}$, where the first uncertainty is statistical and the second is systematic. The result is compatible with the prediction from MC@NLO $\left(25.2_{-6.2}^{+9.6} \mu \mathrm{b}\right)$ and below the prediction from PYTHIA $(49.1 \mu \mathrm{b})$.

In summary, the first measurements of the differential cross sections $d \sigma / d p_{T}^{B}$ and $d \sigma / d y^{B}$ for $B^{0}$ mesons
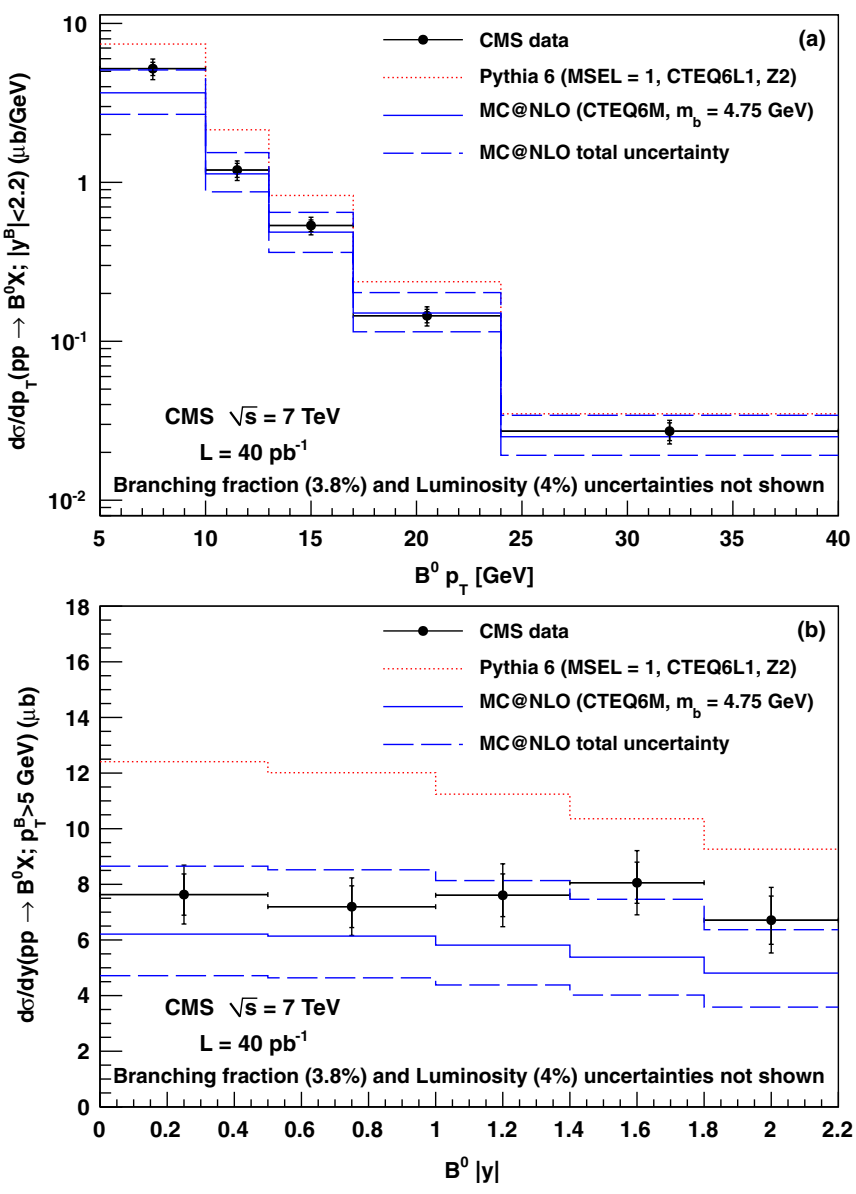

FIG. 2 (color online). Measured differential cross sections (a) $d \sigma / d p_{T}^{B}$ and (b) $d \sigma / d y^{B}$ compared to the theoretical predictions. The inner error bars correspond to the statistical uncertainties and the outer error bars represents the uncorrelated systematic uncertainties added in quadrature to the statistical uncertainties. Overall uncertainties of $4 \%$ for the luminosity and $3.8 \%$ for the branching fractions are not shown. The solid and dashed (blue) lines are the MC@NLO prediction and its uncertainty, respectively. The dotted (red) line is the PYTHIA prediction.

produced in $p p$ collisions at $\sqrt{s}=7 \mathrm{TeV}$ have been presented using the decay $B^{0} \rightarrow J / \psi K_{S}^{0}$. The measurements cover a range in $p_{T}^{B}$ from $5 \mathrm{GeV}$ to more than $30 \mathrm{GeV}$, and the rapidity range $\left|y^{B}\right|<2.2$. The total cross section in this kinematic region lies between the central values of the MC@NLO and PYTHIA predictions, with a rapidity distribution that is flatter than PYTHIA. It is also in agreement within uncertainties with the measured $B^{+}$cross section [9].

We wish to congratulate our colleagues in the CERN accelerator departments for the excellent performance of the LHC machine. We thank the technical and administrative staff at CERN and other CMS institutes, and acknowledge support from: FMSR (Austria); FNRS and FWO (Belgium); CNPq, CAPES, FAPERJ, and FAPESP (Brazil); MES (Bulgaria); CERN; CAS, MoST, and 
NSFC (China); COLCIENCIAS (Colombia); MSES (Croatia); RPF (Cyprus); Academy of Sciences and NICPB (Estonia); Academy of Finland, ME, and HIP (Finland); CEA and CNRS/IN2P3 (France); BMBF, DFG, and HGF (Germany); GSRT (Greece); OTKA and NKTH (Hungary); DAE and DST (India); IPM (Iran); SFI (Ireland); INFN (Italy); NRF and WCU (Korea); LAS (Lithuania); CINVESTAV, CONACYT, SEP, and UASLP-FAI (Mexico); PAEC (Pakistan); SCSR (Poland); FCT (Portugal); JINR (Armenia, Belarus, Georgia, Ukraine, Uzbekistan); MST and MAE (Russia); MSTD (Serbia); MICINN and CPAN (Spain); Swiss Funding Agencies (Switzerland); NSC (Taipei); TUBITAK and TAEK (Turkey); STFC (U.K.); DOE and NSF (U.S.).

[1] C. Albajar et al. (UA1 Collaboration), Phys. Lett. B 213, 405 (1988).

[2] F. Abe et al. (CDF Collaboration), Phys. Rev. Lett. 75, 1451 (1995).

[3] A. Abulencia et al. (CDF Collaboration), Phys. Rev. D 75, 012010 (2007).

[4] S. Abachi et al. (D0 Collaboration), Phys. Rev. Lett. 74, 3548 (1995).

[5] Y. M. Zaitsev (HERA-B Collaboration), Phys. At. Nucl. 72, 675 (2009).

[6] P. Nason, S. Dawson, and R. K. Ellis, Nucl. Phys. B303, 607 (1988)

[7] M. Cacciari et al., J. High Energy Phys. 07 (2004) 033.
[8] CMS Collaboration (CMS Collaboration), J. High Energy Phys. 03 (2011) 090.

[9] CMS Collaboration (CMS Collaboration), Phys. Rev. Lett. 106, 112001 (2011).

[10] R. Aaij et al. (LHCb Collaboration), Phys. Lett. B 694, 209 (2010).

[11] M. Cacciari, M. Greco, and P. Nason, J. High Energy Phys. 05 (1998) 007.

[12] B. A. Kniehl et al., Phys. Rev. D 77, 014011 (2008).

[13] S. Chatrchyan et al. (CMS Collaboration), JINST 3, S08004 (2008).

[14] K. Nakamura et al. (Particle Data Group), J. Phys. G 37, 075021 (2010).

[15] CMS Collaboration (CMS Collaboration), Eur. Phys. J. C 70, 1165 (2010).

[16] T. Sjöstrand, S. Mrenna, and P. Skands, J. High Energy Phys. 05 (2006) 026.

[17] D. J. Lange, Nucl. Instrum. Methods Phys. Res., Sect. A 462, 152 (2001).

[18] S. Agostinelli et al. (GEANT4 Collaboration), Nucl. Instrum. Methods Phys. Res., Sect. A 506, 250 (2003).

[19] CMS Collaboration, Eur. Phys. J. C 71, 1575 (2011).

[20] CMS Collaboration, CMS Physics Analysis Summary Report No. CMS-PAS-TRK-10-002, 2010.

[21] CMS Collaboration, J. High Energy Phys. 05 (2011) 064.

[22] S. Frixione, P. Nason, and B. R. Webber, J. High Energy Phys. 08 (2003) 007.

[23] J. Pumplin et al., J. High Energy Phys. 07 (2002) 012.

[24] R. Field, in Proceedings of the Hadron Collider Physics Symposium 2010, econf C1008233 (2010).

S. Chatrchyan, ${ }^{1}$ V. Khachatryan, ${ }^{1}$ A. M. Sirunyan, ${ }^{1}$ A. Tumasyan, ${ }^{1}$ W. Adam, ${ }^{2}$ T. Bergauer, ${ }^{2}$ M. Dragicevic, ${ }^{2}$ J. Erö, ${ }^{2}$ C. Fabjan, ${ }^{2}$ M. Friedl, ${ }^{2}$ R. Frühwirth, ${ }^{2}$ V. M. Ghete, ${ }^{2}$ J. Hammer, ${ }^{2, b}$ S. Hänsel, ${ }^{2}$ M. Hoch, ${ }^{2}$ N. Hörmann, ${ }^{2}$ J. Hrubec, ${ }^{2}$ M. Jeitler, ${ }^{2}$ G. Kasieczka, ${ }^{2}$ W. Kiesenhofer, ${ }^{2}$ M. Krammer, ${ }^{2}$ D. Liko, ${ }^{2}$ I. Mikulec, ${ }^{2}$ M. Pernicka, ${ }^{2}$ H. Rohringer, ${ }^{2}$ R. Schöfbeck, ${ }^{2}$ J. Strauss, ${ }^{2}$ F. Teischinger, ${ }^{2}$ P. Wagner, ${ }^{2}$ W. Waltenberger, ${ }^{2}$ G. Walzel, ${ }^{2}$ E. Widl, ${ }^{2}$ C.-E. Wulz, ${ }^{2}$ V. Mossolov, ${ }^{3}$ N. Shumeiko, ${ }^{3}$ J. Suarez Gonzalez,${ }^{3}$ L. Benucci, ${ }^{4}$ E. A. De Wolf, ${ }^{4}$ X. Janssen, ${ }^{4}$ T. Maes, ${ }^{4}$

L. Mucibello, ${ }^{4}$ S. Ochesanu, ${ }^{4}$ B. Roland,${ }^{4}$ R. Rougny, ${ }^{4}$ M. Selvaggi, ${ }^{4}$ H. Van Haevermaet, ${ }^{4}$ P. Van Mechelen, ${ }^{4}$ N. Van Remortel, ${ }^{4}$ F. Blekman, ${ }^{5}$ S. Blyweert, ${ }^{5}$ J. D'Hondt, ${ }^{5}$ O. Devroede, ${ }^{5}$ R. Gonzalez Suarez, ${ }^{5}$ A. Kalogeropoulos, ${ }^{5}$ J. Maes, ${ }^{5}$ M. Maes,${ }^{5}$ W. Van Doninck, ${ }^{5}$ P. Van Mulders, ${ }^{5}$ G. P. Van Onsem, ${ }^{5}$ I. Villella, ${ }^{5}$ O. Charaf, ${ }^{6}$ B. Clerbaux, ${ }^{6}$ G. De Lentdecker, ${ }^{6}$ V. Dero, ${ }^{6}$ A. P. R. Gay, ${ }_{6}^{6}$ G. H. Hammad,${ }^{6}$ T. Hreus,${ }^{6}$ P. E. Marage, ${ }^{6}$ L. Thomas, ${ }^{6}$ C. Vander Velde, ${ }^{6}$ P. Vanlaer, ${ }^{6}$ V. Adler, ${ }^{7}$ A. Cimmino, ${ }^{7}$ S. Costantini, ${ }^{7}$ M. Grunewald, ${ }^{7}$ B. Klein,${ }^{7}$ J. Lellouch, ${ }^{7}$ A. Marinov, ${ }^{7}$ J. Mccartin, ${ }^{7}$ D. Ryckbosch, ${ }^{7}$ F. Thyssen, ${ }^{7}$ M. Tytgat, ${ }^{7}$ L. Vanelderen, ${ }^{7}$ P. Verwilligen, ${ }^{7}$ S. Walsh, ${ }^{7}$ N. Zaganidis, ${ }^{7}$ S. Basegmez, ${ }^{8}$ G. Bruno, ${ }^{8}$ J. Caudron,${ }^{8}$ L. Ceard, ${ }^{8}$ E. Cortina Gil,${ }^{8}$ J. De Favereau De Jeneret ${ }^{8}$ C. Delaere, ${ }^{8, b}$ D. Favart, ${ }^{8}$ A. Giammanco, ${ }^{8}$ G. Grégoire, ${ }^{8}$ J. Hollar, ${ }^{8}$ V. Lemaitre, ${ }^{8}$ J. Liao, ${ }^{8}$ O. Militaru, ${ }^{8}$ S. Ovyn, ${ }^{8}$ D. Pagano, ${ }^{8}$ A. Pin,${ }^{8}$ K. Piotrzkowski,${ }^{8}$ N. Schul,${ }^{8}$ N. Beliy, ${ }^{9}$ T. Caebergs, ${ }^{9}$ E. Daubie, ${ }^{9}$ G. A. Alves, ${ }^{10}$ D. De Jesus Damiao, ${ }^{10}$ M. E. Pol, ${ }^{10}$ M. H. G. Souza, ${ }^{10}$ W. Carvalho, ${ }^{11}$ E. M. Da Costa, ${ }^{11}$ C. De Oliveira Martins, ${ }^{11}$ S. Fonseca De Souza, ${ }^{11}$ L. Mundim, ${ }^{11}$ H. Nogima, ${ }^{11}$ V. Oguri, ${ }^{11}$ W. L. Prado Da Silva, ${ }^{11}$ A. Santoro, ${ }^{11}$ S. M. Silva Do Amaral,${ }^{11}$

A. Sznajder ${ }^{11}$ F. Torres Da Silva De Araujo, ${ }^{11}$ F. A. Dias, ${ }^{12}$ T. R. Fernandez Perez Tomei, ${ }^{12}$ E. M. Gregores, ${ }^{12, c}$ C. Lagana, ${ }^{12}$ F. Marinho, ${ }^{12}$ P. G. Mercadante, ${ }^{12, \mathrm{c}}$ S. F. Novaes, ${ }^{12}$ Sandra S. Padula, ${ }^{12}$ N. Darmenov,${ }^{13, b}$ L. Dimitrov, ${ }^{13}$ V. Genchev, ${ }^{13, b}$ P. Iaydjiev, ${ }^{13, b}$ S. Piperov,${ }^{13}$ M. Rodozov, ${ }^{13}$ S. Stoykova, ${ }^{13}$ G. Sultanov, ${ }^{13}$ V. Tcholakov, ${ }^{13}$

R. Trayanov,${ }^{13}$ I. Vankov, ${ }^{13}$ A. Dimitrov, ${ }^{14}$ R. Hadjiiska, ${ }^{14}$ A. Karadzhinova, ${ }^{14}$ V. Kozhuharov,${ }^{14}$ L. Litov,${ }^{14}$ M. Mateev ${ }^{14}$ B. Pavlov, ${ }^{14}$ P. Petkov, ${ }^{14}$ J. G. Bian, ${ }^{15}$ G. M. Chen, ${ }^{15}$ H. S. Chen,${ }^{15}$ C. H. Jiang,${ }^{15}$ D. Liang,${ }^{15}$ S. Liang, ${ }^{15}$ X. Meng, ${ }^{15}$ J. Tao, ${ }^{15}$ J. Wang, ${ }^{15}$ J. Wang, ${ }^{15}$ X. Wang, ${ }^{15}$ Z. Wang,${ }^{15}$ H. Xiao, ${ }^{15}$ M. Xu, ${ }^{15}$ J. Zang, ${ }^{15}$ Z. Zhang, ${ }^{15}$ Y. Ban, ${ }^{16}$ S. Guo, ${ }^{16}$ Y. Guo, ${ }^{16}$ W. Li, ${ }^{16}$ Y. Mao, ${ }^{16}$ S. J. Qian, ${ }^{16}$ H. Teng, ${ }^{16}$ L. Zhang, ${ }^{16}$ B. Zhu, ${ }^{16}$ W. Zou, ${ }^{16}$ 
A. Cabrera, ${ }^{17}$ B. Gomez Moreno, ${ }^{17}$ A. A. Ocampo Rios, ${ }^{17}$ A. F. Osorio Oliveros,${ }^{17}$ J. C. Sanabria, ${ }^{17}$ N. Godinovic,${ }^{18}$ D. Lelas, ${ }^{18}$ K. Lelas ${ }^{18}$ R. Plestina, ${ }^{18, d}$ D. Polic, ${ }^{18}$ I. Puljak, ${ }^{18}$ Z. Antunovic, ${ }^{19}$ M. Dzelalija, ${ }^{19}$ V. Brigljevic,${ }^{20}$ S. Duric,${ }^{20}$ K. Kadija,${ }^{20}$ S. Morovic,${ }^{20}$ A. Attikis,${ }^{21}$ M. Galanti, ${ }^{21}$ J. Mousa,${ }^{21}$ C. Nicolaou, ${ }^{21}$ F. Ptochos,${ }^{21}$ P. A. Razis, ${ }^{21}$ M. Finger, ${ }^{22}$ M. Finger, Jr., ${ }^{22}$ Y. Assran, ${ }^{23, e}$ S. Khalil, ${ }^{23, f}$ M. A. Mahmoud, ${ }^{23, g}$ A. Hektor, ${ }^{24}$ M. Kadastik, ${ }^{24}$ M. Müntel, ${ }^{24}$ M. Raidal, ${ }^{24}$ L. Rebane, ${ }^{24}$ V. Azzolini, ${ }^{25}$ P. Eerola, ${ }^{25}$ G. Fedi, ${ }^{25}$ S. Czellar, ${ }^{26}$ J. Härkönen, ${ }^{26}$ A. Heikkinen, ${ }^{26}$ V. Karimäki, ${ }^{26}$ R. Kinnunen, ${ }^{26}$ M. J. Kortelainen, ${ }^{26}$ T. Lampén, ${ }^{26}$ K. Lassila-Perini, ${ }^{26}$ S. Lehti, ${ }^{26}$ T. Lindén, ${ }^{26}$ P. Luukka, ${ }^{26}$ T. Mäenpää, ${ }^{26}$ E. Tuominen, ${ }^{26}$ J. Tuominiemi, ${ }^{26}$ E. Tuovinen, ${ }^{26}$ D. Ungaro, ${ }^{26}$ L. Wendland, ${ }^{26}$ K. Banzuzi, ${ }^{27}$ A. Korpela, ${ }^{27}$ T. Tuuva, ${ }^{27}$ D. Sillou, ${ }^{28}$ M. Besancon, ${ }^{29}$ S. Choudhury, ${ }^{29}$ M. Dejardin, ${ }^{29}$ D. Denegri, ${ }^{29}$ B. Fabbro, ${ }^{29}$ J. L. Faure,${ }^{29}$ F. Ferri, ${ }^{29}$ S. Ganjour, ${ }^{29}$ F. X. Gentit ${ }^{29}$ A. Givernaud,${ }^{29}$ P. Gras, ${ }^{29}$ G. Hamel de Monchenault, ${ }^{29}$ P. Jarry, ${ }^{29}$ E. Locci, ${ }^{29}$ J. Malcles ${ }^{29}$ M. Marionneau, ${ }^{29}$ L. Millischer, ${ }^{29}$ J. Rander, ${ }^{29}$

A. Rosowsky, ${ }^{29}$ I. Shreyber, ${ }^{29}$ M. Titov, ${ }^{29}$ P. Verrecchia, ${ }^{29}$ S. Baffioni,${ }^{30}$ F. Beaudette, ${ }^{30}$ L. Benhabib,${ }^{30}$

L. Bianchini, ${ }^{30}$ M. Bluj, ${ }^{30, \mathrm{~h}}$ C. Broutin, ${ }^{30}$ P. Busson, ${ }^{30}$ C. Charlot, ${ }^{30}$ T. Dahms, ${ }^{30}$ L. Dobrzynski, ${ }^{30}$ S. Elgammal, ${ }^{30}$ R. Granier de Cassagnac, ${ }^{30}$ M. Haguenauer, ${ }^{30}$ P. Miné, ${ }^{30}$ C. Mironov,${ }^{30}$ C. Ochando, ${ }^{30}$ P. Paganini, ${ }^{30}$ D. Sabes,${ }^{30}$ R. Salerno, ${ }^{30}$ Y. Sirois, ${ }^{30}$ C. Thiebaux,${ }^{30}$ B. Wyslouch, ${ }^{30, i}$ A. Zabi, ${ }^{30}$ J.-L. Agram, ${ }^{31, j}$ J. Andrea, ${ }^{31}$ D. Bloch, ${ }^{31}$ D. Bodin, ${ }^{31}$ J.-M. Brom, ${ }^{31}$ M. Cardaci, ${ }^{31}$ E. C. Chabert, ${ }^{31}$ C. Collard,${ }^{31}$ E. Conte,${ }^{31, j}$ F. Drouhin, ${ }^{31, j}$ C. Ferro, ${ }^{31}$ J.-C. Fontaine, ${ }^{31, j}$ D. Gelé, ${ }^{31}$ U. Goerlach, ${ }^{31}$ S. Greder, ${ }^{31}$ P. Juillot,,${ }^{31}$ M. Karim,,${ }^{31, j}$ A.-C. Le Bihan, ${ }^{31}$ Y. Mikami, ${ }^{31}$ P. Van Hove ${ }^{31}$ F. Fassi, ${ }^{32}$ D. Mercier, ${ }^{32}$ C. Baty,${ }^{33}$ S. Beauceron, ${ }^{33}$ N. Beaupere, ${ }^{33}$ M. Bedjidian, ${ }^{33}$ O. Bondu, ${ }^{33}$ G. Boudoul,${ }^{33}$ D. Boumediene, ${ }^{33}$ H. Brun, ${ }^{33}$ R. Chierici, ${ }^{33}$ D. Contardo, ${ }^{33}$ P. Depasse, ${ }^{33}$ H. El Mamouni, ${ }^{33}$ J. Fay,,${ }^{33}$ S. Gascon, ${ }^{33}$ B. Ille, ${ }^{33}$ T. Kurca, ${ }^{33}$ T. Le Grand,${ }^{33}$ M. Lethuillier, ${ }^{33}$ L. Mirabito, ${ }^{33}$ S. Perries, ${ }^{33}$ V. Sordini, ${ }^{33}$ S. Tosi, ${ }^{33}$ Y. Tschudi, ${ }^{33}$ P. Verdier, ${ }^{33}$ D. Lomidze, ${ }^{34}$ G. Anagnostou, ${ }^{35}$ M. Edelhoff,,${ }^{35}$ L. Feld,,${ }^{35}$ N. Heracleous, ${ }^{35}$ O. Hindrichs,${ }^{35}$ R. Jussen,${ }^{35}$ K. Klein, ${ }^{35}$ J. Merz,${ }^{35}$ N. Mohr,${ }^{35}$ A. Ostapchuk,${ }^{35}$ A. Perieanu, ${ }^{35}$ F. Raupach,${ }^{35}$ J. Sammet, ${ }^{35}$ S. Schael, ${ }^{35}$ D. Sprenger, ${ }^{35}$ H. Weber, ${ }^{35}$ M. Weber, ${ }^{35}$ B. Wittmer, ${ }^{35}$ M. Ata, ${ }^{36}$ W. Bender, ${ }^{36}$ E. Dietz-Laursonn, ${ }^{36}$ M. Erdmann, ${ }^{36}$ J. Frangenheim, ${ }^{36}$ T. Hebbeker, ${ }^{36}$ A. Hinzmann, ${ }^{36}$ K. Hoepfner, ${ }^{36}$ T. Klimkovich, ${ }^{36}$ D. Klingebiel, ${ }^{36}$ P. Kreuzer, ${ }^{36}$ D. Lanske, ${ }^{36, a}$ C. Magass, ${ }^{36}$ M. Merschmeyer ${ }^{36}$ A. Meyer, ${ }^{36}$ P. Papacz, ${ }^{36}$ H. Pieta, ${ }^{36}$ H. Reithler, ${ }^{36}$ S. A. Schmitz,${ }^{36}$ L. Sonnenschein, ${ }^{36}$ J. Steggemann, ${ }^{36}$ D. Teyssier, ${ }^{36}$ M. Tonutti,${ }^{36}$ M. Bontenackels, ${ }^{37}$ M. Davids,${ }^{37}$ M. Duda ${ }^{37}$ G. Flügge,${ }^{37}$ H. Geenen, ${ }^{37}$ M. Giffels, ${ }^{37}$ W. Haj Ahmad ${ }^{37}$ D. Heydhausen, ${ }^{37}$ T. Kress, ${ }^{37}$ Y. Kuessel,,${ }^{37}$ A. Linn, ${ }^{37}$ A. Nowack, ${ }^{37}$ L. Perchalla, ${ }^{37}$ O. Pooth, ${ }^{37}$ J. Rennefeld, ${ }^{37}$ P. Sauerland, ${ }^{37}$ A. Stahl,${ }^{37}$ M. Thomas, ${ }^{37}$ D. Tornier,${ }^{37}$ M. H. Zoeller,${ }^{37}$ M. Aldaya Martin, ${ }^{38}$ W. Behrenhoff, ${ }^{38}$ U. Behrens, ${ }^{38}$ M. Bergholz, ${ }^{38, k}$ K. Borras, ${ }^{38}$ A. Cakir, ${ }^{38}$ A. Campbell, ${ }^{38}$ E. Castro, ${ }^{38}$ D. Dammann, ${ }^{38}$ G. Eckerlin, ${ }^{38}$

D. Eckstein, ${ }^{38}$ A. Flossdorf, ${ }^{38}$ G. Flucke, ${ }^{38}$ A. Geiser, ${ }^{38}$ J. Hauk, ${ }^{38}$ H. Jung,${ }^{38, b}$ M. Kasemann, ${ }^{38}$ I. Katkov, ${ }^{38,1}$ P. Katsas, ${ }^{38}$ C. Kleinwort, ${ }^{38}$ H. Kluge ${ }^{38}$ A. Knutsson, ${ }^{38}$ M. Krämer, ${ }^{38}$ D. Krücker, ${ }^{38}$ E. Kuznetsova, ${ }^{38}$ W. Lange, ${ }^{38}$ W. Lohmann, ${ }^{38, k}$ R. Mankel, ${ }^{38}$ M. Marienfeld, ${ }^{38}$ I.-A. Melzer-Pellmann, ${ }^{38}$ A. B. Meyer, ${ }^{38}$ J. Mnich, ${ }^{38}$

A. Mussgiller, ${ }^{38}$ J. Olzem, ${ }^{38}$ D. Pitzl,${ }^{38}$ A. Raspereza, ${ }^{38}$ A. Raval, ${ }^{38}$ M. Rosin, ${ }^{38}$ R. Schmidt,${ }^{38, k}$ T. Schoerner-Sadenius, ${ }^{38}$ N. Sen,${ }^{38}$ A. Spiridonov, ${ }^{38}$ M. Stein,${ }^{38}$ J. Tomaszewska, ${ }^{38}$ R. Walsh, ${ }^{38}$ C. Wissing,${ }^{38}$ C. Autermann, ${ }^{39}$ V. Blobel, ${ }^{39}$ S. Bobrovskyi, ${ }^{39}$ J. Draeger, ${ }^{39}$ H. Enderle, ${ }^{39}$ U. Gebbert, ${ }^{39}$ K. Kaschube,${ }^{39}$ G. Kaussen, ${ }^{39}$ R. Klanner, ${ }^{39}$ J. Lange,${ }^{39}$ B. Mura ${ }^{39}$ S. Naumann-Emme, ${ }^{39}$ F. Nowak, ${ }^{39}$ N. Pietsch,${ }^{39}$ C. Sander, ${ }^{39}$ H. Schettler, ${ }^{39}$ P. Schleper, ${ }^{39}$ M. Schröder, ${ }^{39}$ T. Schum, ${ }^{39}$ J. Schwandt, ${ }^{39}$ H. Stadie, ${ }^{39}$ G. Steinbrück, ${ }^{39}$ J. Thomsen, ${ }^{39}$

C. Barth, ${ }^{40}$ J. Bauer, ${ }^{40}$ V. Buege,${ }^{40}$ T. Chwalek, ${ }^{40}$ W. De Boer, ${ }^{40}$ A. Dierlamm, ${ }^{40}$ G. Dirkes,${ }^{40}$ M. Feindt,${ }^{40}$

J. Gruschke, ${ }^{40}$ C. Hackstein, ${ }^{40}$ F. Hartmann, ${ }^{40}$ M. Heinrich, ${ }^{40}$ H. Held, ${ }^{40}$ K. H. Hoffmann, ${ }^{40}$ S. Honc,,${ }^{40}$

J. R. Komaragiri, ${ }^{40}$ T. Kuhr, ${ }^{40}$ D. Martschei ${ }^{40}$ S. Mueller ${ }^{40}$ Th. Müller, ${ }^{40}$ M. Niegel, ${ }^{40}$ O. Oberst, ${ }^{40}$ A. Oehler, ${ }^{40}$ J. Ott, ${ }^{40}$ T. Peiffer, ${ }^{40}$ D. Piparo, ${ }^{40}$ G. Quast, ${ }^{40}$ K. Rabbertz, ${ }^{40}$ F. Ratnikov, ${ }^{40}$ N. Ratnikova, ${ }^{40}$ M. Renz, ${ }^{40}$ C. Saout ${ }^{40}$ A. Scheurer, ${ }^{40}$ P. Schieferdecker, ${ }^{40}$ F.-P. Schilling, ${ }^{40}$ M. Schmanau, ${ }^{40}$ G. Schott, ${ }^{40}$ H. J. Simonis, ${ }^{40}$ F. M. Stober, ${ }^{40}$ D. Troendle, ${ }^{40}$ J. Wagner-Kuhr, ${ }^{40}$ T. Weiler ${ }^{40}$ M. Zeise,${ }^{40}$ V. Zhukov, ${ }^{40,1}$ E. B. Ziebarth, ${ }^{40}$ G. Daskalakis,${ }^{41}$

T. Geralis, ${ }^{41}$ K. Karafasoulis, ${ }^{41}$ S. Kesisoglou, ${ }^{41}$ A. Kyriakis, ${ }^{41}$ D. Loukas, ${ }^{41}$ I. Manolakos, ${ }^{41}$ A. Markou, ${ }^{41}$ C. Markou, ${ }^{41}$ C. Mavrommatis, ${ }^{41}$ E. Ntomari, ${ }^{41}$ E. Petrakou, ${ }^{41}$ L. Gouskos, ${ }^{42}$ T. J. Mertzimekis, ${ }^{42}$ A. Panagiotou, ${ }^{42}$ E. Stiliaris, ${ }^{42}$ I. Evangelou, ${ }^{43}$ C. Foudas,${ }^{43}$ P. Kokkas, ${ }^{43}$ N. Manthos, ${ }^{43}$ I. Papadopoulos, ${ }^{43}$ V. Patras, ${ }^{43}$ F. A. Triantis,${ }^{43}$ A. Aranyi,${ }^{44}$ G. Bencze,${ }^{44}$ L. Boldizsar, ${ }^{44}$ C. Hajdu, ${ }^{44, b}$ P. Hidas, ${ }^{44}$ D. Horvath, ${ }^{44, m}$ A. Kapusi, ${ }^{44}$ K. Krajczar, ${ }^{44, n}$ F. Sikler ${ }^{44, b}$ G. I. Veres, ${ }^{44, n}$ G. Vesztergombi, ${ }^{44, n}$ N. Beni, ${ }^{45}$ J. Molnar ${ }^{45}$ J. Palinkas, ${ }^{45}$ Z. Szillasi, ${ }^{45}$ V. Veszpremi ${ }^{45}$ P. Raics, ${ }^{46}$ Z. L. Trocsanyi,${ }^{46}$ B. Ujvari, ${ }^{46}$ S. Bansal, ${ }^{47}$ S. B. Beri, ${ }^{47}$ V. Bhatnagar, ${ }^{47}$ N. Dhingra,${ }^{47}$ R. Gupta ${ }^{47}$ M. Jindal, ${ }^{47}$ M. Kaur, ${ }^{47}$ J. M. Kohli,${ }^{47}$ M.Z. Mehta, ${ }^{47}$ N. Nishu,${ }^{47}$ L. K. Saini ${ }^{47}$ A. Sharma,${ }^{47}$ A. P. Singh, ${ }^{47}$ J. B. Singh, ${ }^{47}$ S. P. Singh,${ }^{47}$ S. Ahuja, ${ }^{48}$ S. Bhattacharya, ${ }^{48}$ B. C. Choudhary, ${ }^{48}$ P. Gupta, ${ }^{48}$ S. Jain, ${ }^{48}$ S. Jain, ${ }^{48}$ 
A. Kumar, ${ }^{48}$ K. Ranjan, ${ }^{48}$ R. K. Shivpuri, ${ }^{48}$ R. K. Choudhury ${ }^{49}$ D. Dutta, ${ }^{49}$ S. Kailas,${ }^{49}$ V. Kumar, ${ }^{49}$ A. K. Mohanty, ${ }^{49, b}$ L. M. Pant ${ }^{49}$ P. Shukla, ${ }^{49}$ T. Aziz, ${ }^{50}$ M. Guchait ${ }^{50, o}$ A. Gurtu, ${ }^{50}$ M. Maity, ${ }^{50, p}$ D. Majumder, ${ }^{50}$ G. Majumder ${ }^{50}$ K. Mazumdar, ${ }^{50}$ G. B. Mohanty, ${ }^{50}$ A. Saha,${ }^{50}$ K. Sudhakar,${ }^{50}$ N. Wickramage,${ }^{50}$ S. Banerjee,${ }^{51}$ S. Dugad, ${ }^{51}$ N. K. Mondal,${ }^{51}$ H. Arfaei, ${ }^{52}$ H. Bakhshiansohi, ${ }^{52, q}$ S. M. Etesami, ${ }^{52}$ A. Fahim,,${ }^{52, q}$ M. Hashemi,${ }^{52}$ A. Jafari, ${ }^{52, \mathrm{q}}$ M. Khakzad, ${ }^{52}$ A. Mohammadi, ${ }^{52, \mathrm{r}}$ M. Mohammadi Najafabadi, ${ }^{52}$ S. Paktinat Mehdiabadi, ${ }^{52}$ B. Safarzadeh ${ }^{52}$ M. Zeinali, ${ }^{52, s}$ M. Abbrescia, ${ }^{53 a, 53 b}$ L. Barbone,${ }^{53 a, 53 b}$ C. Calabria, ${ }^{53 a, 53 b}$ A. Colaleo, ${ }^{53 a}$ D. Creanza, ${ }^{53 a, 53 c}$ N. De Filippis, ${ }^{53 a, 53 c, b}$ M. De Palma, ${ }^{53 a, 53 b}$ L. Fiore, ${ }^{53 a}$ G. Iaselli, ${ }^{53 a, 53 c}$ L. Lusito, ${ }^{53 a, 53 b}$ G. Maggi, ${ }^{53 a, 53 c}$ M. Maggi, ${ }^{53 a}$ N. Manna, ${ }^{53 a, 53 b}$ B. Marangelli, ${ }^{53 a, 53 b}$ S. My, ${ }^{53 a, 53 c}$ S. Nuzzo, ${ }^{53 a, 53 b}$ N. Pacifico, ${ }^{53 a, 53 b}$ G. A. Pierro, ${ }^{53 a}$ A. Pompili, ${ }^{53 a, 53 b}$ G. Pugliese,${ }^{53 a, 53 c}$ F. Romano, ${ }^{53 a, 53 c}$ G. Roselli, ${ }^{53 a, 53 b}$ G. Selvaggi, ${ }^{53 a, 53 b}$ L. Silvestris, ${ }^{53 a}$ R. Trentadue, ${ }^{53 a}$ S. Tupputi, ${ }^{53 a, 53 b}$ G. Zito, ${ }^{53 a}$ G. Abbiendi, ${ }^{54 a}$ A. C. Benvenuti, ${ }^{54 a}$ D. Bonacorsi, ${ }^{54 a}$ S. Braibant-Giacomelli, ${ }^{54 a, 54 b}$ L. Brigliadori, ${ }^{54 a}$ P. Capiluppi, ${ }^{54,54 b}$ A. Castro, ${ }^{54 a, 54 b}$ F. R. Cavallo, ${ }^{54 a}$ M. Cuffiani, ${ }^{54 a, 54 b}$ G. M. Dallavalle, ${ }^{54 a}$ F. Fabbri, ${ }^{54 a}$ A. Fanfani, ${ }^{54 a, 54 b}$ D. Fasanella, ${ }^{54 a}$ P. Giacomelli, ${ }^{54 a}$ M. Giunta, ${ }^{54 a}$ S. Marcellini, ${ }^{54 a}$ G. Masetti, ${ }^{54 a}$ M. Meneghelli, ${ }^{54 a, 54 b}$ A. Montanari, ${ }^{54 a}$ F. L. Navarria, ${ }^{54 a, 54 b}$ F. Odorici, ${ }^{54 a}$ A. Perrotta, ${ }^{54 a}$ F. Primavera, ${ }^{54 a}$ A. M. Rossi, ${ }^{54 a, 54 b}$ T. Rovelli, ${ }^{54 a, 54 b}$ G. Siroli, ${ }^{54 a, 54 b}$ R. Travaglini, ${ }^{54 a, 54 b}$ S. Albergo, ${ }^{55 a, 55 b}$ G. Cappello, ${ }^{55 a, 55 b}$ M. Chiorboli, ${ }^{55 a, 55 b, b}$ S. Costa, ${ }^{55 a, 55 b}$ A. Tricomi, ${ }^{55 a, 55 b}$ C. Tuve, ${ }^{55 \mathrm{a}}$ G. Barbagli, ${ }^{56 \mathrm{a}}$ V. Ciulli, ${ }^{56 \mathrm{a}, 56 \mathrm{~b}}$ C. Civinini, ${ }^{56 \mathrm{a}}$ R. D'Alessandro, ${ }^{56 \mathrm{a}, 56 \mathrm{~b}}$ E. Focardi, ${ }^{56 \mathrm{a}, 56 \mathrm{~b}}$ S. Frosali, ${ }^{56 \mathrm{a}, 56 \mathrm{~b}}$ E. Gallo, ${ }^{56 \mathrm{a}}$ S. Gonzi, ${ }^{56 \mathrm{a}, 56 \mathrm{~b}}$ P. Lenzi, ${ }^{56 \mathrm{a}, 56 \mathrm{~b}}$ M. Meschini, ${ }^{56 \mathrm{a}}$ S. Paoletti, ${ }^{56 \mathrm{a}}$ G. Sguazzoni, ${ }^{56 \mathrm{a}}$ A. Tropiano, ${ }^{56 \mathrm{a}, \mathrm{b}}$ L. Benussi,${ }^{57}$ S. Bianco, ${ }^{57}$ S. Colafranceschi,${ }^{57, t}$ F. Fabbri, ${ }^{57}$ D. Piccolo, ${ }^{57}$ P. Fabbricatore,${ }^{58}$ R. Musenich,${ }^{58}$ A. Benaglia, ${ }^{59 a, 59 b}$ F. De Guio, ${ }^{59 a, 59 b, b}$ L. Di Matteo, ${ }^{59 a, 59 b}$ A. Ghezzi, ${ }^{59 a, 59 b}$ M. Malberti, ${ }^{59 a, 59 b}$ S. Malvezzi, ${ }^{59 a}$ A. Martelli, ${ }^{59 a, 59 b}$ A. Massironi, ${ }^{59 a, 59 b}$ D. Menasce,${ }^{59 a}$ L. Moroni, ${ }^{59 a}$ M. Paganoni, ${ }^{59 a, 59 b}$ D. Pedrini,,${ }^{59 a}$ S. Ragazzi, ${ }^{59 a, 59 b}$ N. Redaelli, ${ }^{59 a}$ S. Sala, ${ }^{59 a}$ T. Tabarelli de Fatis, ${ }^{59 a, 59 b}$ V. Tancini, ${ }^{59 a, 59 b}$ S. Buontempo, ${ }^{60 a}$ C. A. Carrillo Montoya, ${ }^{60 a, b}$ N. Cavallo, ${ }^{60 a, u}$ A. De Cosa,${ }^{60 a, 60 b}$ F. Fabozzi, ${ }^{60 a, u}$ A. O. M. Iorio, ${ }^{60 a, b}$ L. Lista, ${ }^{60 a}$ M. Merola,${ }^{60 a, 60 b}$ P. Paolucci, ${ }^{60 a}$ P. Azzi, ${ }^{61 a}$ N. Bacchetta, ${ }^{61 a}$ P. Bellan,,${ }^{61 a, 61 b}$ D. Bisello, ${ }^{61 a, 61 b}$ A. Branca, ${ }^{61 a}$ R. Carlin, ${ }^{61 \mathrm{a}, 61 \mathrm{~b}}$ P. Checchia, ${ }^{61 \mathrm{a}} \mathrm{M}$. De Mattia, ${ }^{61 \mathrm{a}, 61 \mathrm{~b}}$ T. Dorigo, ${ }^{61 \mathrm{a}}$ U. Dosselli, ${ }^{61 \mathrm{a}}$ F. Fanzago, ${ }^{61 \mathrm{a}} \mathrm{F}$. Gasparini, ${ }^{61 \mathrm{a}, 61 \mathrm{~b}}$ U. Gasparini, ${ }^{61 \mathrm{a}, 61 \mathrm{~b}}$ S. Lacaprara, ${ }^{61 \mathrm{a}}$ I. Lazzizzera, ${ }^{61 \mathrm{a}, 61 \mathrm{c}}$ M. Margoni, ${ }^{61 \mathrm{a}, 61 \mathrm{~b}}$ M. Mazzucato, ${ }^{61 \mathrm{a}}$

A. T. Meneguzzo ${ }^{61 \mathrm{a}, 61 \mathrm{~b}}$ M. Nespolo, ${ }^{61 \mathrm{a}, \mathrm{b}}$ L. Perrozzi, ${ }^{61 \mathrm{a}, \mathrm{b}}$ N. Pozzobon, ${ }^{61 \mathrm{a}, 61 \mathrm{~b}}$ P. Ronchese, ${ }^{61 \mathrm{a}, 61 \mathrm{~b}}$ F. Simonetto, ${ }^{61 \mathrm{a}, 61 \mathrm{~b}}$ E. Torassa, ${ }^{61 \mathrm{a}}$ M. Tosi, ${ }^{61 \mathrm{a}, 61 \mathrm{~b}}$ S. Vanini, ${ }^{61 \mathrm{a}, 61 \mathrm{~b}}$ P. Zotto,${ }^{61 \mathrm{a}, 61 \mathrm{~b}}$ G. Zumerle,${ }^{61 \mathrm{a}, 61 \mathrm{~b}}$ P. Baesso, ${ }^{62 \mathrm{a}, 62 \mathrm{~b}}$ U. Berzano, ${ }^{62 \mathrm{a}}$ S. P. Ratti, ${ }^{62 a, 62 b}$ C. Riccardi, ${ }^{62 a, 62 b}$ P. Torre, ${ }^{62 a, 62 b}$ P. Vitulo, ${ }^{62 a, 62 b}$ C. Viviani, ${ }^{62 a, 62 b}$ M. Biasini, ${ }^{63 a, 63 b}$ G. M. Bilei, ${ }^{63 a}$ B. Caponeri, ${ }^{63 a, 63 b}$ L. Fanò, ${ }^{63 a, 63 b}$ P. Lariccia, ${ }^{63 a, 63 b}$ A. Lucaroni, ${ }^{63 a, 63 b, b}$ G. Mantovani, ${ }^{63 a, 63 b}$ M. Menichelli, ${ }^{63 a}$ A. Nappi ${ }^{63 a, 63 b}$ F. Romeo, ${ }^{63 a, 63 b}$ A. Santocchia ${ }^{63 a, 63 b}$ S. Taroni, ${ }^{63 a, 63 b, b}$ M. Valdata, ${ }^{63 a, 63 b}$ P. Azzurri, ${ }^{64 a, 64 c}$ G. Bagliesi, ${ }^{64 a}$ J. Bernardini, ${ }^{64 a, 64 b}$ T. Boccali, ${ }^{64 a, b}$ G. Broccolo, ${ }^{64 a, 64 c}$ R. Castaldi, ${ }^{64 a}$ R. T. D'Agnolo, ${ }^{64 a, 64 c}$ R. Dell'Orso, ${ }^{64 a}$ F. Fiori, ${ }^{64 a, 64 b}$ L. Foà, ${ }^{64 a, 64 c}$ A. Giassi, ${ }^{64 a}$ A. Kraan, ${ }^{64 a}$ F. Ligabue, ${ }^{64 a, 64 c}$ T. Lomtadze, ${ }^{64 a}$ L. Martini, ${ }^{64 a, v}$ A. Messineo, ${ }^{64 a, 64 b}$ F. Palla, ${ }^{64 a}$ G. Segneri, ${ }^{64 a}$ A. T. Serban,${ }^{64 a}$ P. Spagnolo, ${ }^{64 a}$ R. Tenchini, ${ }^{64 a}$ G. Tonelli, ${ }^{64 a, 64 b, b}$ A. Venturi, ${ }^{64 a, b}$ P. G. Verdini, ${ }^{64 a}$ L. Barone, ${ }^{65 a, 65 b}$ F. Cavallari, ${ }^{65 a}$ D. Del Re, ${ }^{65 a, 65 b}$ E. Di Marco, ${ }^{65 a, 65 b}$ M. Diemoz, ${ }^{65 a}$ D. Franci, ${ }^{65 a, 65 b}$ M. Grassi, ${ }^{65 a, b}$ E. Longo,${ }^{65 a, 65 b}$ S. Nourbakhsh, ${ }^{65 a}$ G. Organtini, ${ }^{65 a, 65 b}$ F. Pandolfi, ${ }^{65 a, 65 b, b}$ R. Paramatti, ${ }^{65 a}$ S. Rahatlou, ${ }^{65 a, 65 b}$ N. Amapane, ${ }^{66 a, 66 b}$ R. Arcidiacono, ${ }^{66 a, 66 c}$ S. Argiro, ${ }^{66 a, 66 b}$ M. Arneodo, ${ }^{66 a, 66 c}$ C. Biino, ${ }^{66 \mathrm{a}}$ C. Botta,${ }^{66 a, 66 b, b}$ N. Cartiglia, ${ }^{66 \mathrm{a}}$ R. Castello, ${ }^{66 \mathrm{a}, 66 \mathrm{~b}} \mathrm{M}$. Costa, ${ }^{66 \mathrm{a}, 66 \mathrm{~b}}$ N. Demaria, ${ }^{66 \mathrm{a}}$ A. Graziano, ${ }^{66 \mathrm{a}, 66 \mathrm{~b}, \mathrm{~b}}$ C. Mariotti, ${ }^{66 \mathrm{a}}$ M. Marone,${ }^{66 \mathrm{a}, 66 \mathrm{~b}}$ S. Maselli, ${ }^{66 \mathrm{a}}$ E. Migliore, ${ }^{66 \mathrm{a}, 66 \mathrm{~b}}$ G. Mila, ${ }^{66 \mathrm{a}, 66 \mathrm{~b}}$ V. Monaco, ${ }^{66 a, 66 b}$ M. Musich, ${ }^{66 a, 66 b}$ M. M. Obertino, ${ }^{66 a, 66 c}$ N. Pastrone, ${ }^{66 a}$ M. Pelliccioni, ${ }^{66 a, 66 b}$ A. Romero, ${ }^{66 a, 66 b}$ M. Ruspa, ${ }^{66 a, 66 c}$ R. Sacchi, ${ }^{66 a, 66 b}$ V. Sola ${ }^{66 a, 66 b}$ A. Solano, ${ }^{66 a, 66 b}$ A. Staiano, ${ }^{66 a}$ A. Vilela Pereira, ${ }^{66 a}$ S. Belforte,${ }^{67 a}$ F. Cossutti, ${ }^{67 a}$ G. Della Ricca, ${ }^{67 a, 67 b}$ B. Gobbo, ${ }^{67 a}$ D. Montanino, ${ }^{67 a, 67 b}$ A. Penzo, ${ }^{67 a}$ S. G. Heo, ${ }^{68}$ S. K. Nam,${ }^{68}$ S. Chang, ${ }^{69}$ J. Chung, ${ }^{69}$ D. H. Kim, ${ }^{69}$ G. N. Kim, ${ }^{69}$ J. E. Kim, ${ }^{69}$ D. J. Kong, ${ }^{69}$ H. Park, ${ }^{69}$ S. R. Ro, ${ }^{69}$ D. Son ${ }^{69}$ D. C. Son, ${ }^{69}$ T. Son, ${ }^{69}$ Zero Kim, ${ }^{70}$ J. Y. Kim, ${ }^{70}$ S. Song, ${ }^{70}$ S. Choi, ${ }^{71}$ B. Hong, ${ }^{71}$ M. S. Jeong, ${ }^{71}$ M. Jo, ${ }^{71}$ H. Kim,${ }^{71}$ J. H. Kim, ${ }^{71}$ T. J. Kim, ${ }^{71}$ K. S. Lee, ${ }^{71}$ D. H. Moon, ${ }^{71}$ S. K. Park, ${ }^{71}$ H. B. Rhee, ${ }^{71}$ E. Seo, ${ }^{71}$ S. Shin, ${ }^{71}$ K. S. Sim, ${ }^{71}$ M. Choi ${ }^{72}$ S. Kang, ${ }^{72}$ H. Kim, ${ }^{72}$ C. Park, ${ }^{72}$ I. C. Park,${ }^{72}$ S. Park, ${ }^{72}$ G. Ryu,${ }^{72}$ Y. Choi, ${ }^{73}$ Y. K. Choi, ${ }^{73}$ J. Goh, ${ }^{73}$ M. S. Kim, ${ }^{73}$ E. Kwon, ${ }^{73}$ J. Lee, ${ }^{73}$ S. Lee, ${ }^{73}$ H. Seo, ${ }^{73}$ I. Yu, ${ }^{73}$ M. J. Bilinskas, ${ }^{74}$ I. Grigelionis,${ }^{74}$ M. Janulis, ${ }^{74}$ D. Martisiute, ${ }^{74}$ P. Petrov, ${ }^{74}$ T. Sabonis, ${ }^{74}$ H. Castilla-Valdez, ${ }^{75}$ E. De La Cruz-Burelo, ${ }^{75}$ R. Lopez-Fernandez,${ }^{75}$

R. Magaña Villalba, ${ }^{75}$ A. Sánchez-Hernández, ${ }^{75}$ L. M. Villasenor-Cendejas ${ }^{75}$ S. Carrillo Moreno, ${ }^{76}$

F. Vazquez Valencia ${ }^{76}$ H. A. Salazar Ibarguen, ${ }^{77}$ E. Casimiro Linares,${ }^{78}$ A. Morelos Pineda,${ }^{78}$ M. A. Reyes-Santos, ${ }^{78}$ D. Krofcheck ${ }^{79}$ J. Tam, ${ }^{79}$ P. H. Butler, ${ }^{80}$ R. Doesburg, ${ }^{80}$ H. Silverwood, ${ }^{80}$ M. Ahmad, ${ }^{81}$ I. Ahmed, ${ }^{81}$ M. I. Asghar, ${ }^{81}$ H. R. Hoorani, ${ }^{81}$ W. A. Khan, ${ }^{81}$ T. Khurshid, ${ }^{81}$ S. Qazi, ${ }^{81}$ M. Cwiok, ${ }^{82}$ W. Dominik, ${ }^{82}$ K. Doroba, ${ }^{82}$ A. Kalinowski, ${ }^{82}$ 
M. Konecki, ${ }^{82}$ J. Krolikowski, ${ }^{82}$ T. Frueboes, ${ }^{83}$ R. Gokieli, ${ }^{83}$ M. Górski, ${ }^{83}$ M. Kazana, ${ }^{83}$ K. Nawrocki, ${ }^{83}$ K. Romanowska-Rybinska, ${ }^{83}$ M. Szleper ${ }^{83}$ G. Wrochna ${ }^{83}$ P. Zalewski ${ }^{83}$ N. Almeida,${ }^{84}$ P. Bargassa, ${ }^{84}$ A. David ${ }^{84}$ P. Faccioli, ${ }^{84}$ P. G. Ferreira Parracho, ${ }^{84}$ M. Gallinaro,${ }^{84}$ P. Musella, ${ }^{84}$ A. Nayak, ${ }^{84}$ P. Q. Ribeiro,${ }^{84}$ J. Seixas,${ }^{84}$ J. Varela, ${ }^{84}$ S. Afanasiev, ${ }^{85}$ I. Belotelov, ${ }^{85}$ P. Bunin, ${ }^{85}$ I. Golutvin, ${ }^{85}$ A. Kamenev, ${ }^{85}$ V. Karjavin, ${ }^{85}$ G. Kozlov, ${ }^{85}$ A. Lanev, ${ }^{85}$ P. Moisenz, ${ }^{85}$ V. Palichik, ${ }^{85}$ V. Perelygin, ${ }^{85}$ S. Shmatov, ${ }^{85}$ V. Smirnov, ${ }^{85}$ A. Volodko, ${ }^{85}$ A. Zarubin,${ }^{85}$ V. Golovtsov, ${ }^{86}$ Y. Ivanov, ${ }^{86}$ V. Kim, ${ }^{86}$ P. Levchenko, ${ }^{86}$ V. Murzin, ${ }^{86}$ V. Oreshkin, ${ }^{86}$ I. Smirnov, ${ }^{86}$ V. Sulimov, ${ }^{86}$ L. Uvarov, ${ }^{86}$ S. Vavilov, ${ }^{86}$ A. Vorobyev, ${ }^{86}$ A. Vorobyev, ${ }^{86}$ Yu. Andreev, ${ }^{87}$ A. Dermenev, ${ }^{87}$ S. Gninenko, ${ }^{87}$ N. Golubev, ${ }^{87}$ M. Kirsanov,${ }^{87}$ N. Krasnikov,${ }^{87}$ V. Matveev, ${ }^{87}$ A. Pashenkov, ${ }^{87}$ A. Toropin, ${ }^{87}$ S. Troitsky, ${ }^{87}$ V. Epshteyn, ${ }^{88}$ V. Gavrilov, ${ }^{88}$ V. Kaftanov, ${ }^{88, a}$ M. Kossov, ${ }^{88, b}$ A. Krokhotin, ${ }^{88}$ N. Lychkovskaya, ${ }^{88}$ V. Popov, ${ }^{88}$ G. Safronov, ${ }^{88}$ S. Semenov, ${ }^{88}$ V. Stolin, ${ }^{88}$ E. Vlasov, ${ }^{88}$ A. Zhokin, ${ }^{88}$ E. Boos, ${ }^{89}$ M. Dubinin,${ }^{89, w}$ L. Dudko, ${ }^{89}$ A. Ershov, ${ }^{89}$ A. Gribushin, ${ }^{89}$ O. Kodolova, ${ }^{89}$ I. Lokhtin, ${ }^{89}$ A. Markina, ${ }^{89}$ S. Obraztsov, ${ }^{89}$ M. Perfilov, ${ }^{89}$ S. Petrushanko, ${ }^{89}$ L. Sarycheva, ${ }^{89}$ V. Savrin, ${ }^{89}$ A. Snigirev, ${ }^{89}$ V. Andreev, ${ }^{90}$ M. Azarkin, ${ }^{90}$ I. Dremin,,${ }^{90}$ M. Kirakosyan, ${ }^{90}$ A. Leonidov, ${ }^{90}$ S. V. Rusakov, ${ }^{90}$ A. Vinogradov,${ }^{90}$ I. Azhgirey, ${ }^{91}$ S. Bitioukov,${ }^{91}$ V. Grishin, ${ }^{91, b}$ V. Kachanov, ${ }^{91}$ D. Konstantinov, ${ }^{91}$ A. Korablev, ${ }^{91}$ V. Krychkine, ${ }^{91}$ V. Petrov, ${ }^{91}$ R. Ryutin, ${ }^{91}$ S. Slabospitsky, ${ }^{91}$

A. Sobol,${ }^{91}$ L. Tourtchanovitch, ${ }^{91}$ S. Troshin,${ }^{91}$ N. Tyurin,,${ }^{91}$ A. Uzunian, ${ }^{91}$ A. Volkov, ${ }^{91}$ P. Adzic, ${ }^{92, x}$ M. Djordjevic, ${ }^{92}$ D. Krpic, ${ }^{92, x}$ J. Milosevic,${ }^{92}$ M. Aguilar-Benitez, ${ }^{93}$ J. Alcaraz Maestre, ${ }^{93}$ P. Arce, ${ }^{93}$ C. Battilana, ${ }^{93}$ E. Calvo, ${ }^{93}$ M. Cepeda, ${ }^{93}$ M. Cerrada, ${ }^{93}$ M. Chamizo Llatas, ${ }^{93}$ N. Colino, ${ }^{93}$ B. De La Cruz,${ }^{93}$ A. Delgado Peris, ${ }^{93}$ C. Diez Pardos, ${ }^{93}$ D. Domínguez Vázquez, ${ }^{93}$ C. Fernandez Bedoya, ${ }^{93}$ J. P. Fernández Ramos, ${ }^{93}$ A. Ferrando, ${ }^{93}$ J. Flix, ${ }^{93}$ M. C. Fouz, ${ }^{93}$ P. Garcia-Abia, ${ }^{93}$ O. Gonzalez Lopez,${ }^{93}$ S. Goy Lopez,${ }^{93}$ J. M. Hernandez, ${ }^{93}$ M. I. Josa, ${ }^{93}$ G. Merino, ${ }^{93}$ J. Puerta Pelayo, ${ }^{93}$ I. Redondo, ${ }^{93}$ L. Romero, ${ }^{93}$ J. Santaolalla, ${ }^{93}$ M. S. Soares, ${ }^{93}$ C. Willmott, ${ }^{93}$ C. Albajar, ${ }^{94}$ G. Codispoti, ${ }^{94}$ J. F. de Trocóniz, ${ }^{94}$ J. Cuevas, ${ }^{95}$ J. Fernandez Menendez, ${ }^{95}$ S. Folgueras, ${ }^{95}$ I. Gonzalez Caballero, ${ }^{95}$ L. Lloret Iglesias, ${ }^{95}$ J. M. Vizan Garcia, ${ }^{95}$ J. A. Brochero Cifuentes, ${ }^{96}$ I. J. Cabrillo, ${ }^{96}$ A. Calderon, ${ }^{96}$ S. H. Chuang, ${ }^{96}$ J. Duarte Campderros, ${ }^{96}$ M. Felcini,,${ }^{96, y}$ M. Fernandez, ${ }^{96}$ G. Gomez, ${ }^{96}$ J. Gonzalez Sanchez, ${ }^{96}$ C. Jorda, ${ }^{96}$ P. Lobelle Pardo, ${ }^{96}$ A. Lopez Virto, ${ }^{96}$ J. Marco, ${ }^{96}$ R. Marco, ${ }^{96}$ C. Martinez Rivero, ${ }^{96}$ F. Matorras, ${ }^{96}$ F. J. Munoz Sanchez,${ }^{96}$ J. Piedra Gomez, ${ }^{96, z}$ T. Rodrigo, ${ }^{96}$ A. Y. Rodríguez-Marrero, ${ }^{96}$ A. Ruiz-Jimeno, ${ }^{96}$ L. Scodellaro, ${ }^{96}$ M. Sobron Sanudo, ${ }^{96}$ I. Vila, ${ }^{96}$ R. Vilar Cortabitarte, ${ }^{96}$ D. Abbaneo, ${ }^{97}$ E. Auffray, ${ }^{97}$ G. Auzinger, ${ }^{97}$ P. Baillon, ${ }^{97}$ A. H. Ball, ${ }^{97}$ D. Barney, ${ }^{97}$ A. J. Bell,,${ }^{97, \text { aa }}$ D. Benedetti, ${ }^{97}$ C. Bernet, ${ }^{97, d}$ W. Bialas,,${ }^{97}$ P. Bloch,${ }^{97}$ A. Bocci, ${ }^{97}$ S. Bolognesi, ${ }^{97}$ M. Bona, ${ }^{97}$ H. Breuker, ${ }^{97}$ G. Brona, ${ }^{97}$ K. Bunkowski,${ }^{97}$ T. Camporesi, ${ }^{97}$ G. Cerminara, ${ }^{97}$ J. A. Coarasa Perez, ${ }^{97}$ B. Curé,${ }^{97}$ D. D'Enterria, ${ }^{97}$ A. De Roeck, ${ }^{97}$ S. Di Guida, ${ }^{97}$ A. Elliott-Peisert, ${ }^{97}$ B. Frisch, ${ }^{97}$ W. Funk, ${ }^{97}$ A. Gaddi, ${ }^{97}$ S. Gennai, ${ }^{97}$ G. Georgiou, ${ }^{97}$ H. Gerwig, ${ }^{97}$ D. Gigi, ${ }^{97}$ K. Gill,,${ }^{97}$ D. Giordano, ${ }^{97}$ F. Glege,${ }^{97}$ R. Gomez-Reino Garrido, ${ }^{97}$ M. Gouzevitch, ${ }^{97}$ P. Govoni, ${ }^{97}$ S. Gowdy, ${ }^{97}$ L. Guiducci, ${ }^{97}$ M. Hansen,${ }^{97}$ C. Hartl, ${ }^{97}$ J. Harvey, ${ }^{97}$ J. Hegeman, ${ }^{97}$ B. Hegner, ${ }^{97}$ H. F. Hoffmann, ${ }^{97}$ A. Honma, ${ }^{97}$ V. Innocente, ${ }^{97}$ P. Janot,${ }^{97}$ K. Kaadze, ${ }^{97}$ E. Karavakis, ${ }^{97}$ P. Lecoq, ${ }^{97}$ C. Lourenço, ${ }^{97}$ T. Mäki, ${ }^{97}$ L. Malgeri,,${ }^{97}$ M. Mannelli, ${ }^{97}$ L. Masetti, ${ }^{97}$ A. Maurisset, ${ }^{97}$ F. Meijers,${ }^{97}$ S. Mersi,${ }^{97}$ E. Meschi, ${ }^{97}$ R. Moser,${ }^{97}$ M. U. Mozer, ${ }^{97}$ M. Mulders,${ }^{97}$ E. Nesvold,,${ }^{97, b}$ M. Nguyen, ${ }^{97}$ T. Orimoto, ${ }^{97}$ L. Orsini, ${ }^{97}$ E. Perez, ${ }^{97}$ A. Petrilli, ${ }^{97}$ A. Pfeiffer,${ }^{97}$ M. Pierini, ${ }^{97}$ M. Pimiä, ${ }^{97}$ G. Polese, ${ }^{97}$ A. Racz,${ }^{97}$ J. Rodrigues Antunes, ${ }^{97}$ G. Rolandi, ${ }^{97, b b}$ T. Rommerskirchen,${ }^{97}$ C. Rovelli, ${ }^{97, c c}$ M. Rovere,${ }^{97}$ H. Sakulin, ${ }^{97}$ C. Schäfer, ${ }^{97}$ C. Schwick, ${ }^{97}$ I. Segoni, ${ }^{97}$ A. Sharma,,${ }^{97}$ P. Siegrist, ${ }^{97}$ M. Simon, ${ }^{97}$ P. Sphicas, ${ }^{97, d d}$ M. Spiropulu, ${ }^{97, w}$ M. Stoye, ${ }^{97}$ P. Tropea, ${ }^{97}$

A. Tsirou, ${ }^{97}$ P. Vichoudis, ${ }^{97}$ M. Voutilainen, ${ }^{97}$ W. D. Zeuner,${ }^{97}$ W. Bertl,${ }^{98}$ K. Deiters, ${ }^{98}$ W. Erdmann,,${ }^{98}$

K. Gabathuler, ${ }^{98}$ R. Horisberger, ${ }^{98}$ Q. Ingram, ${ }^{98}$ H. C. Kaestli, ${ }^{98}$ S. König, ${ }^{98}$ D. Kotlinski, ${ }^{98}$ U. Langenegger, ${ }^{98}$ F. Meier, ${ }^{98}$ D. Renker, ${ }^{98}$ T. Rohe, ${ }^{98}$ J. Sibille, ${ }^{98, \text { ee }}$ A. Starodumov, ${ }^{98, f f}$ P. Bortignon, ${ }^{99}$ L. Caminada, ${ }^{99, \text { gg }}$ N. Chanon, ${ }^{99}$ Z. Chen, ${ }^{99}$ S. Cittolin, ${ }^{99}$ G. Dissertori, ${ }^{99}$ M. Dittmar, ${ }^{99}$ J. Eugster, ${ }^{99}$ K. Freudenreich,${ }^{99}$ C. Grab, ${ }^{99}$ A. Hervé,,${ }^{99}$ W. Hintz, ${ }^{99}$ P. Lecomte, ${ }^{99}$ W. Lustermann, ${ }^{99}$ C. Marchica, ${ }^{99, g g}$ P. Martinez Ruiz del Arbol,,${ }^{99}$ P. Meridiani, ${ }^{99}$ P. Milenovic, ${ }^{99, \text { hh }}$ F. Moortgat, ${ }^{99}$ C. Nägeli, ${ }^{99, g g}$ P. Nef, ${ }^{99}$ F. Nessi-Tedaldi, ${ }^{99}$ L. Pape,${ }^{99}$ F. Pauss, ${ }^{99}$ T. Punz, ${ }^{99}$ A. Rizzi, ${ }^{99}$ F. J. Ronga, ${ }^{99}$ M. Rossini,${ }^{99}$ L. Sala, ${ }^{99}$ A. K. Sanchez,${ }^{99}$ M.-C. Sawley, ${ }^{99}$ B. Stieger, ${ }^{99}$ L. Tauscher, ${ }^{99,}$

A. Thea ${ }^{99}$ K. Theofilatos, ${ }^{99}$ D. Treille, ${ }^{99}$ C. Urscheler, ${ }^{99}$ R. Wallny, ${ }^{99}$ M. Weber, ${ }^{99}$ L. Wehrli, ${ }^{99}$ J. Weng,${ }^{99}$ E. Aguiló, ${ }^{100}$ C. Amsler, ${ }^{100}$ V. Chiochia, ${ }^{100}$ S. De Visscher, ${ }^{100}$ C. Favaro, ${ }^{100}$ M. Ivova Rikova, ${ }^{100}$ B. Millan Mejias, ${ }^{100}$ P. Otiougova, ${ }^{100}$ C. Regenfus, ${ }^{100}$ P. Robmann, ${ }^{100}$ A. Schmidt, ${ }^{100}$ H. Snoek, ${ }^{100}$ Y. H. Chang,,${ }^{101}$ K. H. Chen, ${ }^{101}$ S. Dutta, ${ }^{101}$ C. M. Kuo,${ }^{101}$ S. W. Li, ${ }^{101}$ W. Lin,${ }^{101}$ Z. K. Liu,${ }^{101}$ Y. J. Lu, ${ }^{101}$ D. Mekterovic,${ }^{101}$ R. Volpe,${ }^{101}$ J. H. Wu, ${ }^{101}$ S. S. Yu, ${ }^{101}$ P. Bartalini, ${ }^{102}$ P. Chang, ${ }^{102}$ Y. H. Chang, ${ }^{102}$ Y. W. Chang, ${ }^{102}$ Y. Chao, ${ }^{102}$ K. F. Chen, ${ }^{102}$ W.-S. Hou, ${ }^{102}$ Y. Hsiung, ${ }^{102}$ K. Y. Kao, ${ }^{102}$ Y. J. Lei, ${ }^{102}$ R.-S. Lu, ${ }^{102}$ J. G. Shiu, ${ }^{102}$ Y. M. Tzeng, ${ }^{102}$ M. Wang, ${ }^{102}$ A. Adiguzel,,${ }^{103}$ 
M. N. Bakirci, ${ }^{103, \text { ii }}$ S. Cerci, ${ }^{103, j j}$ C. Dozen, ${ }^{103}$ I. Dumanoglu, ${ }^{103}$ E. Eskut, ${ }^{103}$ S. Girgis, ${ }^{103}$ G. Gokbulut, ${ }^{103}$ Y. Guler, ${ }^{103}$ E. Gurpinar, ${ }^{103}$ I. Hos, ${ }^{103}$ E. E. Kangal, ${ }^{103}$ T. Karaman, ${ }^{103}$ A. Kayis Topaksu, ${ }^{103}$ A. Nart, ${ }^{103}$ G. Onengut, ${ }^{103}$ K. Ozdemir, ${ }^{103}$ S. Ozturk, ${ }^{103}$ A. Polatoz, ${ }^{103}$ K. Sogut, ${ }^{103, k \mathrm{kk}}$ D. Sunar Cerci, ${ }^{103, j \mathrm{jj}}$ B. Tali, ${ }^{103}$ H. Topakli, ${ }^{103, \text { ii }}$ D. Uzun, ${ }^{103}$ L. N. Vergili, ${ }^{103}$ M. Vergili, ${ }^{103}$ C. Zorbilmez, ${ }^{103}$ I. V. Akin, ${ }^{104}$ T. Aliev, ${ }^{104}$ S. Bilmis, ${ }^{104}$ M. Deniz, ${ }^{104}$ H. Gamsizkan, ${ }^{104}$ A. M. Guler, ${ }^{104}$ K. Ocalan, ${ }^{104}$ A. Ozpineci, ${ }^{104}$ M. Serin, ${ }^{104}$ R. Sever, ${ }^{104}$ U.E. Surat, ${ }^{104}$ E. Yildirim, ${ }^{104}$ M. Zeyrek, ${ }^{104}$ M. Deliomeroglu, ${ }^{105}$ D. Demir,,${ }^{105,11}$ E. Gülmez, ${ }^{105}$ B. Isildak, ${ }^{105}$ M. Kaya, ${ }^{105, \mathrm{~mm}}$ O. Kaya, ${ }^{105, \mathrm{~mm}}$ S. Ozkorucuklu, ${ }^{105, \mathrm{nn}}$ N. Sonmez, ${ }^{105, \mathrm{oo}}$ L. Levchuk, ${ }^{106}$ F. Bostock, ${ }^{107}$ J. J. Brooke, ${ }^{107}$ T. L. Cheng, ${ }^{107}$ E. Clement,${ }^{107}$ D. Cussans, ${ }^{107}$ R. Frazier, ${ }^{107}$ J. Goldstein, ${ }^{107}$ M. Grimes, ${ }^{107}$ M. Hansen,,${ }^{107}$ D. Hartley, ${ }^{107}$ G. P. Heath, ${ }^{107}$ H. F. Heath,,${ }^{107}$ J. Jackson, ${ }^{107}$ L. Kreczko, ${ }^{107}$ S. Metson, ${ }^{107}$ D. M. Newbold,,${ }^{107, p p}$ K. Nirunpong, ${ }^{107}$ A. Poll, ${ }^{107}$ S. Senkin, ${ }^{107}$ V. J. Smith, ${ }^{107}$ S. Ward,${ }^{107}$ L. Basso, ${ }^{108, q q}$ K. W. Bell, ${ }^{108}$ A. Belyaev, ${ }^{108, q q}$ C. Brew, ${ }^{108}$ R. M. Brown, ${ }^{108}$ B. Camanzi, ${ }^{108}$ D. J. A. Cockerill, ${ }^{108}$ J. A. Coughlan, ${ }^{108}$ K. Harder, ${ }^{108}$ S. Harper, ${ }^{108}$

B. W. Kennedy, ${ }^{108}$ E. Olaiya, ${ }^{108}$ D. Petyt,${ }^{108}$ B. C. Radburn-Smith, ${ }^{108}$ C. H. Shepherd-Themistocleous, ${ }^{108}$ I. R. Tomalin, ${ }^{108}$ W. J. Womersley, ${ }^{108}$ S. D. Worm, ${ }^{108}$ R. Bainbridge, ${ }^{109}$ G. Ball, ${ }^{109}$ J. Ballin, ${ }^{109}$ R. Beuselinck, ${ }^{109}$ O. Buchmuller, ${ }^{109}$ D. Colling, ${ }^{109}$ N. Cripps,${ }^{109}$ M. Cutajar, ${ }^{109}$ G. Davies, ${ }^{109}$ M. Della Negra, ${ }^{109}$ W. Ferguson,,${ }^{109}$ J. Fulcher, ${ }^{109}$ D. Futyan, ${ }^{109}$ A. Gilbert, ${ }^{109}$ A. Guneratne Bryer, ${ }^{109}$ G. Hall, ${ }^{109}$ Z. Hatherell,,${ }^{109}$ J. Hays, ${ }^{109}$ G. Iles,${ }^{109}$ M. Jarvis, ${ }^{109}$ G. Karapostoli, ${ }^{109}$ L. Lyons, ${ }^{109}$ B. C. MacEvoy, ${ }^{109}$ A.-M. Magnan, ${ }^{109}$ J. Marrouche,${ }^{109}$ B. Mathias, ${ }^{109}$ R. Nandi, ${ }^{109}$ J. Nash, ${ }^{109}$ A. Nikitenko, ${ }^{109, f f}$ A. Papageorgiou, ${ }^{109}$ M. Pesaresi,${ }^{109}$ K. Petridis, ${ }^{109}$ M. Pioppi,${ }^{109, \text { rr }}$

D. M. Raymond, ${ }^{109}$ S. Rogerson, ${ }^{109}$ N. Rompotis, ${ }^{109}$ A. Rose, ${ }^{109}$ M. J. Ryan, ${ }^{109}$ C. Seez, ${ }^{109}$ P. Sharp, ${ }^{109}$

A. Sparrow, ${ }^{109}$ A. Tapper, ${ }^{109}$ S. Tourneur, ${ }^{109}$ M. Vazquez Acosta, ${ }^{109}$ T. Virdee,${ }^{109}$ S. Wakefield, ${ }^{109}$ N. Wardle, ${ }^{109}$

D. Wardrope ${ }^{109}$ T. Whyntie, ${ }^{109}$ M. Barrett, ${ }^{110}$ M. Chadwick,${ }^{110}$ J.E. Cole, ${ }^{110}$ P. R. Hobson, ${ }^{110}$ A. Khan, ${ }^{110}$

P. Kyberd, ${ }^{110}$ D. Leslie, ${ }^{110}$ W. Martin, ${ }^{110}$ I. D. Reid, ${ }^{110}$ L. Teodorescu, ${ }^{110}$ K. Hatakeyama, ${ }^{111}$ T. Bose,${ }^{112}$ E. Carrera Jarrin, ${ }^{112}$ C. Fantasia, ${ }^{112}$ A. Heister, ${ }^{112}$ J. St. John, ${ }^{112}$ P. Lawson, ${ }^{112}$ D. Lazic, ${ }^{112}$ J. Rohlf, ${ }^{112}$ D. Sperka, ${ }^{112}$

L. Sulak, ${ }^{112}$ A. Avetisyan, ${ }^{113}$ S. Bhattacharya, ${ }^{113}$ J.P. Chou, ${ }^{113}$ D. Cutts, ${ }^{113}$ A. Ferapontov, ${ }^{113}$ U. Heintz, ${ }^{113}$ S. Jabeen, ${ }^{113}$ G. Kukartsev, ${ }^{113}$ G. Landsberg, ${ }^{113}$ M. Narain, ${ }^{113}$ D. Nguyen,,${ }^{113}$ M. Segala, ${ }^{113}$ T. Sinthuprasith, ${ }^{113}$ T. Speer, ${ }^{113}$ K. V. Tsang, ${ }^{113}$ R. Breedon, ${ }^{114}$ M. Calderon De La Barca Sanchez, ${ }^{114}$ S. Chauhan, ${ }^{114}$ M. Chertok, ${ }^{114}$ J. Conway, ${ }^{114}$ P. T. Cox, ${ }^{114}$ J. Dolen, ${ }^{114}$ R. Erbacher, ${ }^{114}$ E. Friis, ${ }^{114}$ W. Ko, ${ }^{114}$ A. Kopecky, ${ }^{114}$ R. Lander, ${ }^{114}$ H. Liu, ${ }^{114}$ S. Maruyama, ${ }^{114}$ T. Miceli, ${ }^{114}$ M. Nikolic, ${ }^{114}$ D. Pellett,,${ }^{114}$ J. Robles, ${ }^{114}$ S. Salur, ${ }^{114}$ T. Schwarz, ${ }^{114}$ M. Searle, ${ }^{114}$ J. Smith, ${ }^{114}$ M. Squires, ${ }^{114}$ M. Tripathi, ${ }^{114}$ R. Vasquez Sierra, ${ }^{114}$ C. Veelken, ${ }^{114}$ V. Andreev, ${ }^{115}$ K. Arisaka, ${ }^{115}$ D. Cline,${ }^{115}$ R. Cousins,${ }^{115}$ A. Deisher, ${ }^{115}$ J. Duris, ${ }^{115}$ S. Erhan, ${ }^{115}$ C. Farrell,${ }^{115}$ J. Hauser, ${ }^{115}$ M. Ignatenko, ${ }^{115}$ C. Jarvis, ${ }^{115}$ C. Plager,${ }^{115}$ G. Rakness,${ }^{115}$ P. Schlein,,${ }^{15, a}$ J. Tucker, ${ }^{115}$ V. Valuev,${ }^{115}$ J. Babb,${ }^{116}$ A. Chandra, ${ }^{116}$ R. Clare, ${ }^{116}$ J. Ellison, ${ }^{116}$ J. W. Gary, ${ }^{116}$ F. Giordano, ${ }^{116}$ G. Hanson, ${ }^{116}$ G. Y. Jeng, ${ }^{116}$ S. C. Kao, ${ }^{116}$ F. Liu, ${ }^{116}$ H. Liu, ${ }^{116}$ O. R. Long, ${ }^{116}$ A. Luthra, ${ }^{116}$ H. Nguyen, ${ }^{116}$ B. C. Shen, ${ }^{116, a}$ R. Stringer, ${ }^{116}$ J. Sturdy,,${ }^{116}$ S. Sumowidagdo, ${ }^{116}$ R. Wilken, ${ }^{116}$ S. Wimpenny, ${ }^{116}$ W. Andrews, ${ }^{117}$ J. G. Branson, ${ }^{117}$ G. B. Cerati,,${ }^{117}$ E. Dusinberre, ${ }^{117}$ D. Evans, ${ }^{117}$ F. Golf,${ }^{117}$ A. Holzner,${ }^{117}$ R. Kelley, ${ }^{117}$ M. Lebourgeois,,${ }^{117}$ J. Letts, ${ }^{117}$ B. Mangano, ${ }^{117}$ S. Padhi, ${ }^{117}$ C. Palmer, ${ }^{117}$ G. Petrucciani, ${ }^{117}$ H. Pi, ${ }^{117}$ M. Pieri, ${ }^{117}$ R. Ranieri, ${ }^{117}$ M. Sani, ${ }^{117}$ V. Sharma, ${ }^{117}$ S. Simon, ${ }^{117}$ Y. Tu, ${ }^{117}$ A. Vartak, ${ }^{117}$ S. Wasserbaech, ${ }^{117, s s}$ F. Würthwein, ${ }^{117}$ A. Yagil, ${ }^{117}$ J. Yoo, ${ }^{117}$ D. Barge, ${ }^{118}$ R. Bellan, ${ }^{118}$ C. Campagnari, ${ }^{118}$ M. D'Alfonso, ${ }^{118}$ T. Danielson, ${ }^{118}$ K. Flowers, ${ }^{118}$ P. Geffert,,${ }^{118}$ J. Incandela, ${ }^{118}$ C. Justus, ${ }^{118}$ P. Kalavase, ${ }^{118}$ S. A. Koay, ${ }^{118}$ D. Kovalskyi,,${ }^{18}$ V. Krutelyov, ${ }^{118}$ S. Lowette, ${ }^{118}$ N. Mccoll, ${ }^{118}$ V. Pavlunin, ${ }^{118}$ F. Rebassoo, ${ }^{118}$ J. Ribnik, ${ }^{118}$ J. Richman, ${ }^{118}$ R. Rossin, ${ }^{118}$ D. Stuart,${ }^{118}$ W. To, ${ }^{118}$ J. R. Vlimant, ${ }^{118}$ A. Apresyan, ${ }^{119}$ A. Bornheim, ${ }^{119}$ J. Bunn, ${ }^{119}$ Y. Chen, ${ }^{119}$ M. Gataullin, ${ }^{119}$ Y. Ma, ${ }^{119}$ A. Mott, ${ }^{119}$ H. B. Newman, ${ }^{119}$ C. Rogan, ${ }^{119}$ K. Shin, ${ }^{119}$ V. Timciuc, ${ }^{119}$ P. Traczyk, ${ }^{119}$ J. Veverka, ${ }^{119}$ R. Wilkinson, ${ }^{119}$ Y. Yang, ${ }^{119}$

R. Y. Zhu, ${ }^{119}$ B. Akgun, ${ }^{120}$ R. Carroll, ${ }^{120}$ T. Ferguson, ${ }^{120}$ Y. Iiyama, ${ }^{120}$ D. W. Jang, ${ }^{120}$ S. Y. Jun, ${ }^{120}$ Y. F. Liu, ${ }^{120}$ M. Paulini, ${ }^{120}$ J. Russ, ${ }^{120}$ H. Vogel,${ }^{120}$ I. Vorobiev, ${ }^{120}$ J. P. Cumalat, ${ }^{121}$ M. E. Dinardo, ${ }^{121}$ B. R. Drell, ${ }^{121}$ C. J. Edelmaier ${ }^{121}$ W. T. Ford, ${ }^{121}$ A. Gaz,${ }^{121}$ B. Heyburn, ${ }^{121}$ E. Luiggi Lopez,${ }^{121}$ U. Nauenberg, ${ }^{121}$ J. G. Smith, ${ }^{121}$ K. Stenson, ${ }^{121}$ K. A. Ulmer, ${ }^{121}$ S. R. Wagner, ${ }^{121}$ S. L. Zang, ${ }^{121}$ L. Agostino, ${ }^{122}$ J. Alexander, ${ }^{122}$ D. Cassel,${ }^{122}$ A. Chatterjee, ${ }^{122}$ S. Das, ${ }^{122}$ N. Eggert, ${ }^{122}$ L. K. Gibbons, ${ }^{122}$ B. Heltsley, ${ }^{122}$ W. Hopkins, ${ }^{122}$ A. Khukhunaishvili, ${ }^{122}$ B. Kreis, ${ }^{122}$ G. Nicolas Kaufman, ${ }^{122}$ J. R. Patterson, ${ }^{122}$ D. Puigh, ${ }^{122}$ A. Ryd, ${ }^{122}$ E. Salvati, ${ }^{122}$ X. Shi, ${ }^{122}$ W. Sun, ${ }^{122}$ W. D. Teo, ${ }^{122}$ J. Thom, ${ }^{122}$ J. Thompson, ${ }^{122}$ J. Vaughan, ${ }^{122}$ Y. Weng,,${ }^{122}$ L. Winstrom, ${ }^{122}$ P. Wittich, ${ }^{122}$ A. Biselli, ${ }^{123}$ G. Cirino, ${ }^{123}$ D. Winn, ${ }^{123}$ S. Abdullin, ${ }^{124}$ M. Albrow, ${ }^{124}$ J. Anderson, ${ }^{124}$ G. Apollinari, ${ }^{124}$ M. Atac,,${ }^{124}$

J. A. Bakken, ${ }^{124}$ S. Banerjee, ${ }^{124}$ L. A. T. Bauerdick, ${ }^{124}$ A. Beretvas, ${ }^{124}$ J. Berryhill, ${ }^{124}$ P. C. Bhat, ${ }^{124}$ I. Bloch, ${ }^{124}$ F. Borcherding, ${ }^{124}$ K. Burkett, ${ }^{124}$ J. N. Butler, ${ }^{124}$ V. Chetluru, ${ }^{124}$ H. W. K. Cheung, ${ }^{124}$ F. Chlebana, ${ }^{124}$ S. Cihangir, ${ }^{124}$ 
W. Cooper, ${ }^{124}$ D. P. Eartly, ${ }^{124}$ V. D. Elvira, ${ }^{124}$ S. Esen, ${ }^{124}$ I. Fisk, ${ }^{124}$ J. Freeman, ${ }^{124}$ Y. Gao, ${ }^{124}$ E. Gottschalk, ${ }^{124}$

D. Green, ${ }^{124}$ K. Gunthoti, ${ }^{124}$ O. Gutsche, ${ }^{124}$ J. Hanlon, ${ }^{124}$ R. M. Harris, ${ }^{124}$ J. Hirschauer, ${ }^{124}$ B. Hooberman, ${ }^{124}$ H. Jensen, ${ }^{124}$ M. Johnson, ${ }^{124}$ U. Joshi, ${ }^{124}$ R. Khatiwada, ${ }^{124}$ B. Klima, ${ }^{124}$ K. Kousouris, ${ }^{124}$ S. Kunori, ${ }^{124}$ S. Kwan, ${ }^{124}$ C. Leonidopoulos, ${ }^{124}$ P. Limon, ${ }^{124}$ D. Lincoln, ${ }^{124}$ R. Lipton, ${ }^{124}$ J. Lykken, ${ }^{124}$ K. Maeshima, ${ }^{124}$ J. M. Marraffino, ${ }^{124}$ D. Mason, ${ }^{124}$ P. McBride, ${ }^{124}$ T. Miao, ${ }^{124}$ K. Mishra, ${ }^{124}$ S. Mrenna, ${ }^{124}$ Y. Musienko, ${ }^{124, t t}$ C. Newman-Holmes, ${ }^{124}$ V. O'Dell, ${ }^{124}$ R. Pordes, ${ }^{124}$ O. Prokofyev, ${ }^{124}$ N. Saoulidou, ${ }^{124}$ E. Sexton-Kennedy, ${ }^{124}$ S. Sharma, ${ }^{124}$ A. Soha, ${ }^{124}$ W. J. Spalding, ${ }^{124}$ L. Spiegel, ${ }^{124}$ P. Tan, ${ }^{124}$ L. Taylor, ${ }^{124}$ S. Tkaczyk, ${ }^{124}$ L. Uplegger, ${ }^{124}$ E. W. Vaandering, ${ }^{124}$ R. Vidal, ${ }^{124}$ J. Whitmore, ${ }^{124}$ W. Wu, ${ }^{124}$ F. Yang, ${ }^{124}$ F. Yumiceva, ${ }^{124}$ J. C. Yun, ${ }^{124}$ D. Acosta, ${ }^{125}$ P. Avery, ${ }^{125}$ D. Bourilkov, ${ }^{125}$ M. Chen, ${ }^{125}$ M. De Gruttola, ${ }^{125}$ G. P. Di Giovanni, ${ }^{125}$ D. Dobur, ${ }^{125}$ A. Drozdetskiy, ${ }^{125}$ R. D. Field, ${ }^{125}$ M. Fisher, ${ }^{125}$ Y. Fu, ${ }^{125}$ I. K. Furic, ${ }^{125}$ J. Gartner, ${ }^{125}$ B. Kim, ${ }^{125}$ J. Konigsberg, ${ }^{125}$ A. Korytov, ${ }^{125}$

A. Kropivnitskaya, ${ }^{125}$ T. Kypreos, ${ }^{125}$ K. Matchev, ${ }^{125}$ G. Mitselmakher, ${ }^{125}$ L. Muniz, ${ }^{125}$ Y. Pakhotin, ${ }^{125}$ C. Prescott, ${ }^{125}$ R. Remington, ${ }^{125}$ M. Schmitt, ${ }^{125}$ B. Scurlock, ${ }^{125}$ P. Sellers, ${ }^{125}$ N. Skhirtladze, ${ }^{125}$ M. Snowball, ${ }^{125}$ D. Wang, ${ }^{125}$ J. Yelton, ${ }^{125}$ M. Zakaria, ${ }^{125}$ C. Ceron, ${ }^{126}$ V. Gaultney, ${ }^{126}$ L. Kramer, ${ }^{126}$ L. M. Lebolo, ${ }^{126}$ S. Linn, ${ }^{126}$ P. Markowitz, ${ }^{126}$ G. Martinez, ${ }^{126}$ D. Mesa, ${ }^{126}$ J. L. Rodriguez, ${ }^{126}$ T. Adams, ${ }^{127}$ A. Askew, ${ }^{127}$ D. Bandurin, ${ }^{127}$ J. Bochenek, ${ }^{127}$ J. Chen, ${ }^{127}$ B. Diamond, ${ }^{127}$ S. V. Gleyzer, ${ }^{127}$ J. Haas, ${ }^{127}$ S. Hagopian, ${ }^{127}$ V. Hagopian, ${ }^{127}$ M. Jenkins, ${ }^{127}$ K. F. Johnson, ${ }^{127}$ H. Prosper, ${ }^{127}$ L. Quertenmont, ${ }^{127}$ S. Sekmen, ${ }^{127}$ V. Veeraraghavan, ${ }^{127}$ M. M. Baarmand, ${ }^{128}$ B. Dorney, ${ }^{128}$ S. Guragain, ${ }^{128}$ M. Hohlmann, ${ }^{128}$ H. Kalakhety, ${ }^{128}$ R. Ralich, ${ }^{128}$ I. Vodopiyanov, ${ }^{128}$ M. R. Adams, ${ }^{129}$ I. M. Anghel, ${ }^{129}$ L. Apanasevich, ${ }^{129}$ Y. Bai, ${ }^{129}$ V. E. Bazterra, ${ }^{129}$ R. R. Betts, ${ }^{129}$ J. Callner, ${ }^{129}$ R. Cavanaugh, ${ }^{129}$ C. Dragoiu, ${ }^{129}$ L. Gauthier, ${ }^{129}$ C. E. Gerber, ${ }^{129}$ D. J. Hofman, ${ }^{129}$ S. Khalatyan, ${ }^{129}$ G. J. Kunde, ${ }^{129}$ F. Lacroix, ${ }^{129}$ M. Malek, ${ }^{129}$ C. O’Brien, ${ }^{129}$ C. Silvestre, ${ }^{129}$ A. Smoron, ${ }^{129}$ D. Strom, ${ }^{129}$ N. Varelas, ${ }^{129}$ U. Akgun, ${ }^{130}$ E. A. Albayrak, ${ }^{130}$ B. Bilki, ${ }^{130}$ W. Clarida, ${ }^{130}$ F. Duru, ${ }^{130}$ C. K. Lae, ${ }^{130}$ E. McCliment, ${ }^{130}$ J.-P. Merlo, ${ }^{130}$ H. Mermerkaya, ${ }^{130, \text { uu }}$ A. Mestvirishvili, ${ }^{130}$ A. Moeller, ${ }^{130}$ J. Nachtman, ${ }^{130}$ C. R. Newsom, ${ }^{130}$ E. Norbeck, ${ }^{130}$ J. Olson, ${ }^{130}$ Y. Onel, ${ }^{130}$ F. Ozok, ${ }^{130}$ S. Sen, ${ }^{130}$ J. Wetzel, ${ }^{130}$ T. Yetkin, ${ }^{130}$ K. Yi, ${ }^{130}$ B. A. Barnett, ${ }^{131}$ B. Blumenfeld, ${ }^{131}$ A. Bonato, ${ }^{131}$ C. Eskew, ${ }^{131}$ D. Fehling, ${ }^{131}$ G. Giurgiu, ${ }^{131}$ A. V. Gritsan, ${ }^{131}$ Z. J. Guo, ${ }^{131}$ G. Hu, ${ }^{131}$ P. Maksimovic, ${ }^{131}$ S. Rappoccio, ${ }^{131}$ M. Swartz, ${ }^{131}$ N. V. Tran, ${ }^{131}$ A. Whitbeck, ${ }^{131}$ P. Baringer, ${ }^{132}$ A. Bean, ${ }^{132}$ G. Benelli, ${ }^{132}$ O. Grachov, ${ }^{132}$ R. P. Kenny Iii, ${ }^{132}$ M. Murray, ${ }^{132}$ D. Noonan, ${ }^{132}$ S. Sanders, ${ }^{132}$ J. S. Wood, ${ }^{132}$ V. Zhukova, ${ }^{132}$ A. f. Barfuss, ${ }^{133}$ T. Bolton, ${ }^{133}$ I. Chakaberia, ${ }^{133}$ A. Ivanov, ${ }^{133}$ S. Khalil, ${ }^{133}$ M. Makouski, ${ }^{133}$ Y. Maravin, ${ }^{133}$ S. Shrestha, ${ }^{133}$ I. Svintradze, ${ }^{133}$ Z. Wan, ${ }^{133}$ J. Gronberg, ${ }^{134}$ D. Lange, ${ }^{134}$ D. Wright ${ }^{134}$ A. Baden, ${ }^{135}$ M. Boutemeur, ${ }^{135}$ S. C. Eno, ${ }^{135}$ D. Ferencek, ${ }^{135}$ J. A. Gomez, ${ }^{135}$ N. J. Hadley, ${ }^{135}$ R. G. Kellogg, ${ }^{135}$ M. Kirn, ${ }^{135}$

Y. Lu, ${ }^{135}$ A. C. Mignerey, ${ }^{135}$ K. Rossato, ${ }^{135}$ P. Rumerio, ${ }^{135}$ F. Santanastasio, ${ }^{135}$ A. Skuja, ${ }^{135}$ J. Temple, ${ }^{135}$ M. B. Tonjes, ${ }^{135}$ S. C. Tonwar, ${ }^{135}$ E. Twedt, ${ }^{135}$ B. Alver, ${ }^{136}$ G. Bauer, ${ }^{136}$ J. Bendavid, ${ }^{136}$ W. Busza, ${ }^{136}$ E. Butz, ${ }^{136}$ I. A. Cali, ${ }^{136}$ M. Chan, ${ }^{136}$ V. Dutta, ${ }^{136}$ P. Everaerts, ${ }^{136}$ G. Gomez Ceballos, ${ }^{136}$ M. Goncharov, ${ }^{136}$ K. A. Hahn, ${ }^{136}$ P. Harris, ${ }^{136}$ Y. Kim, ${ }^{136}$ M. Klute, ${ }^{136}$ Y.-J. Lee, ${ }^{136}$ W. Li, ${ }^{136}$ C. Loizides, ${ }^{136}$ P. D. Luckey, ${ }^{136}$ T. Ma, ${ }^{136}$ S. Nahn, ${ }^{136}$ C. Paus, ${ }^{136}$ D. Ralph, ${ }^{136}$ C. Roland, ${ }^{136}$ G. Roland, ${ }^{136}$ M. Rudolph, ${ }^{136}$ G. S. F. Stephans, ${ }^{136}$ F. Stöckli, ${ }^{136}$ K. Sumorok, ${ }^{136}$ K. Sung, ${ }^{136}$ E. A. Wenger, ${ }^{136}$ S. Xie, ${ }^{136}$ M. Yang, ${ }^{136}$ Y. Yilmaz, ${ }^{136}$ A. S. Yoon, ${ }^{136}$ M. Zanetti, ${ }^{136}$ P. Cole, ${ }^{137}$ S. I. Cooper, ${ }^{137}$ P. Cushman, ${ }^{137}$ B. Dahmes, ${ }^{137}$ A. De Benedetti, ${ }^{137}$ P. R. Dudero, ${ }^{137}$ G. Franzoni, ${ }^{137}$

J. Haupt, ${ }^{137}$ K. Klapoetke, ${ }^{137}$ Y. Kubota, ${ }^{137}$ J. Mans, ${ }^{137}$ V. Rekovic, ${ }^{137}$ R. Rusack, ${ }^{137}$ M. Sasseville, ${ }^{137}$ A. Singovsky, ${ }^{137}$ L. M. Cremaldi, ${ }^{138}$ R. Godang, ${ }^{138}$ R. Kroeger, ${ }^{138}$ L. Perera, ${ }^{138}$ R. Rahmat, ${ }^{138}$ D. A. Sanders, ${ }^{138}$ D. Summers, ${ }^{138}$ K. Bloom, ${ }^{139}$ S. Bose, ${ }^{139}$ J. Butt, ${ }^{139}$ D. R. Claes, ${ }^{139}$ A. Dominguez, ${ }^{139}$ M. Eads, ${ }^{139}$ J. Keller, ${ }^{139}$

T. Kelly, ${ }^{139}$ I. Kravchenko, ${ }^{139}$ J. Lazo-Flores, ${ }^{139}$ H. Malbouisson, ${ }^{139}$ S. Malik, ${ }^{139}$ G. R. Snow, ${ }^{139}$ U. Baur, ${ }^{140}$ A. Godshalk, ${ }^{140}$ I. Iashvili, ${ }^{140}$ S. Jain, ${ }^{140}$ A. Kharchilava, ${ }^{140}$ A. Kumar, ${ }^{140}$ S. P. Shipkowski, ${ }^{140}$ K. Smith, ${ }^{140}$ G. Alverson, ${ }^{141}$ E. Barberis, ${ }^{141}$ D. Baumgartel, ${ }^{141}$ O. Boeriu, ${ }^{141}$ M. Chasco, ${ }^{141}$ S. Reucroft, ${ }^{141}$ J. Swain, ${ }^{141}$ D. Trocino, ${ }^{141}$ D. Wood, ${ }^{141}$ J. Zhang, ${ }^{141}$ A. Anastassov, ${ }^{142}$ A. Kubik, ${ }^{142}$ N. Odell, ${ }^{142}$ R. A. Ofierzynski, ${ }^{142}$ B. Pollack, ${ }^{142}$ A. Pozdnyakov, ${ }^{142}$ M. Schmitt, ${ }^{142}$ S. Stoynev, ${ }^{142}$ M. Velasco, ${ }^{142}$ S. Won, ${ }^{142}$ L. Antonelli, ${ }^{143}$ D. Berry, ${ }^{143}$ M. Hildreth, ${ }^{143}$ C. Jessop, ${ }^{143}$ D. J. Karmgard, ${ }^{143}$ J. Kolb, ${ }^{143}$ T. Kolberg, ${ }^{143}$ K. Lannon, ${ }^{143}$ W. Luo, ${ }^{143}$ S. Lynch, ${ }^{143}$ N. Marinelli, ${ }^{143}$ D. M. Morse, ${ }^{143}$ T. Pearson, ${ }^{143}$ R. Ruchti, ${ }^{143}$ J. Slaunwhite, ${ }^{143}$ N. Valls, ${ }^{143}$ M. Wayne, ${ }^{143}$ J. Ziegler, ${ }^{143}$ B. Bylsma, ${ }^{144}$ L. S. Durkin, ${ }^{144}$ J. Gu, ${ }^{144}$ C. Hill, ${ }^{144}$ P. Killewald, ${ }^{144}$ K. Kotov, ${ }^{144}$ T. Y. Ling, ${ }^{144}$ M. Rodenburg, ${ }^{144}$ G. Williams, ${ }^{144}$ N. Adam, ${ }^{145}$ E. Berry, ${ }^{145}$ P. Elmer, ${ }^{145}$ D. Gerbaudo, ${ }^{145}$ V. Halyo, ${ }^{145}$ P. Hebda, ${ }^{145}$ A. Hunt, ${ }^{145}$ J. Jones, ${ }^{145}$ E. Laird, ${ }^{145}$ D. Lopes Pegna, ${ }^{145}$ D. Marlow, ${ }^{145}$ T. Medvedeva, ${ }^{145}$ M. Mooney, ${ }^{145}$ J. Olsen, ${ }^{145}$ P. Piroué, ${ }^{145}$ X. Quan, ${ }^{145}$ H. Saka, ${ }^{145}$ D. Stickland, ${ }^{145}$ C. Tully, ${ }^{145}$ J. S. Werner, ${ }^{145}$ A. Zuranski, ${ }^{145}$ J. G. Acosta, ${ }^{146}$ X. T. Huang, ${ }^{146}$ A. Lopez, ${ }^{146}$ H. Mendez, ${ }^{146}$ S. Oliveros, ${ }^{146}$ J. E. Ramirez Vargas, ${ }^{146}$ 
A. Zatserklyaniy, ${ }^{146}$ E. Alagoz, ${ }^{147}$ V. E. Barnes, ${ }^{147}$ G. Bolla, ${ }^{147}$ L. Borrello, ${ }^{147}$ D. Bortoletto, ${ }^{147}$ A. Everett, ${ }^{147}$ A. F. Garfinkel, ${ }^{147}$ L. Gutay, ${ }^{147}$ Z. Hu, ${ }^{147}$ M. Jones, ${ }^{147}$ O. Koybasi, ${ }^{147}$ M. Kress, ${ }^{147}$ A. T. Laasanen, ${ }^{147}$ N. Leonardo, ${ }^{147}$ C. Liu, ${ }^{147}$ V. Maroussov, ${ }^{147}$ P. Merkel, ${ }^{147}$ D. H. Miller, ${ }^{147}$ N. Neumeister, ${ }^{147}$ I. Shipsey, ${ }^{147}$ D. Silvers, ${ }^{147}$ A. Svyatkovskiy, ${ }^{147}$ H. D. Yoo, ${ }^{147}$ J. Zablocki, ${ }^{147}$ Y. Zheng, ${ }^{147}$ P. Jindal, ${ }^{148}$ N. Parashar, ${ }^{148}$ C. Boulahouache, ${ }^{149}$ V. Cuplov, ${ }^{149}$ K. M. Ecklund, ${ }^{149}$ F. J. M. Geurts, ${ }^{149}$ B. P. Padley, ${ }^{149}$ R. Redjimi, ${ }^{149}$ J. Roberts, ${ }^{149}$ J. Zabel, ${ }^{149}$ B. Betchart, ${ }^{150}$ A. Bodek, ${ }^{150}$ Y. S. Chung, ${ }^{150}$ R. Covarelli, ${ }^{150}$ P. de Barbaro, ${ }^{150}$ R. Demina, ${ }^{150}$ Y. Eshaq, ${ }^{150}$ H. Flacher, ${ }^{150}$ A. Garcia-Bellido, ${ }^{150}$ P. Goldenzweig, ${ }^{150}$ Y. Gotra, ${ }^{150}$ J. Han, ${ }^{150}$ A. Harel, ${ }^{150}$ D. C. Miner, ${ }^{150}$ D. Orbaker, ${ }^{150}$ G. Petrillo, ${ }^{150}$ D. Vishnevskiy, ${ }^{150}$ M. Zielinski, ${ }^{150}$ A. Bhatti, ${ }^{151}$ R. Ciesielski, ${ }^{151}$ L. Demortier, ${ }^{151}$ K. Goulianos, ${ }^{151}$ G. Lungu, ${ }^{151}$ S. Malik, ${ }^{151}$ C. Mesropian, ${ }^{151}$ M. Yan, ${ }^{151}$ O. Atramentov, ${ }^{152}$ A. Barker, ${ }^{152}$ D. Duggan, ${ }^{152}$ Y. Gershtein, ${ }^{152}$ R. Gray, ${ }^{152}$ E. Halkiadakis, ${ }^{152}$ D. Hidas, ${ }^{152}$ D. Hits, ${ }^{152}$ A. Lath, ${ }^{152}$ S. Panwalkar, ${ }^{152}$ R. Patel, ${ }^{152}$ A. Richards, ${ }^{152}$ K. Rose, ${ }^{152}$ S. Schnetzer, ${ }^{152}$ S. Somalwar, ${ }^{152}$ R. Stone, ${ }^{152}$ S. Thomas, ${ }^{152}$ G. Cerizza, ${ }^{153}$ M. Hollingsworth, ${ }^{153}$ S. Spanier, ${ }^{153}$ Z. C. Yang, ${ }^{153}$ A. York, ${ }^{153}$ J. Asaadi, ${ }^{154}$ R. Eusebi, ${ }^{154}$ J. Gilmore, ${ }^{154}$ A. Gurrola, ${ }^{154}$ T. Kamon, ${ }^{154}$ V. Khotilovich, ${ }^{154}$ R. Montalvo, ${ }^{154}$ C. N. Nguyen, ${ }^{154}$ I. Osipenkov, ${ }^{154}$ J. Pivarski, ${ }^{154}$ A. Safonov, ${ }^{154}$ S. Sengupta, ${ }^{154}$ A. Tatarinov, ${ }^{154}$ D. Toback, ${ }^{154}$ M. Weinberger, ${ }^{154}$ N. Akchurin, ${ }^{155}$ C. Bardak, ${ }^{155}$ J. Damgov, ${ }^{155}$ C. Jeong, ${ }^{155}$ K. Kovitanggoon, ${ }^{155}$ S. W. Lee, ${ }^{155}$ Y. Roh, ${ }^{155}$ A. Sill, ${ }^{155}$ I. Volobouev, ${ }^{155}$ R. Wigmans, ${ }^{155}$ E. Yazgan, ${ }^{155}$ E. Appelt, ${ }^{156}$ E. Brownson, ${ }^{156}$ D. Engh, ${ }^{156}$ C. Florez, ${ }^{156}$ W. Gabella, ${ }^{156}$ M. Issah, ${ }^{156}$ W. Johns, ${ }^{156}$ P. Kurt, ${ }^{156}$ C. Maguire, ${ }^{156}$ A. Melo, ${ }^{156}$ P. Sheldon, ${ }^{156}$ B. Snook, ${ }^{156}$ S. Tuo, ${ }^{156}$ J. Velkovska, ${ }^{156}$ M. W. Arenton, ${ }^{157}$ M. Balazs, ${ }^{157}$ S. Boutle, ${ }^{157}$ B. Cox,${ }^{157}$ B. Francis, ${ }^{157}$ R. Hirosky, ${ }^{157}$ A. Ledovskoy, ${ }^{157}$ C. Lin, ${ }^{157}$ C. Neu, ${ }^{157}$ R. Yohay, ${ }^{157}$ S. Gollapinni, ${ }^{158}$ R. Harr, ${ }^{158}$ P. E. Karchin, ${ }^{158}$ P. Lamichhane, ${ }^{158}$ M. Mattson, ${ }^{158}$ C. Milstène, ${ }^{158}$ A. Sakharov, ${ }^{158}$ M. Anderson, ${ }^{159}$ M. Bachtis, ${ }^{159}$ J. N. Bellinger, ${ }^{159}$ D. Carlsmith, ${ }^{159}$ S. Dasu, ${ }^{159}$ J. Efron, ${ }^{159}$ K. Flood, ${ }^{159}$ L. Gray, ${ }^{159}$ K. S. Grogg, ${ }^{159}$ M. Grothe, ${ }^{159}$ R. Hall-Wilton, ${ }^{159}$ M. Herndon, ${ }^{159}$ P. Klabbers, ${ }^{159}$ J. Klukas, ${ }^{159}$ A. Lanaro, ${ }^{159}$ C. Lazaridis, ${ }^{159}$ J. Leonard, ${ }^{159}$ R. Loveless, ${ }^{159}$ A. Mohapatra, ${ }^{159}$ F. Palmonari, ${ }^{159}$ D. Reeder, ${ }^{159}$ I. Ross, ${ }^{159}$ A. Savin, ${ }^{159}$ W. H. Smith, ${ }^{159}$ J. Swanson, ${ }^{159}$ and M. Weinberg ${ }^{159}$

\title{
(CMS Collaboration)
}

\author{
${ }^{1}$ Yerevan Physics Institute, Yerevan, Armenia \\ ${ }^{2}$ Institut für Hochenergiephysik der OeAW, Wien, Austria \\ ${ }^{3}$ National Centre for Particle and High Energy Physics, Minsk, Belarus \\ ${ }^{4}$ Universiteit Antwerpen, Antwerpen, Belgium \\ ${ }^{5}$ Vrije Universiteit Brussel, Brussel, Belgium \\ ${ }^{6}$ Université Libre de Bruxelles, Bruxelles, Belgium \\ ${ }^{7}$ Ghent University, Ghent, Belgium \\ ${ }^{8}$ Université Catholique de Louvain, Louvain-la-Neuve, Belgium \\ ${ }^{9}$ Université de Mons, Mons, Belgium \\ ${ }^{10}$ Centro Brasileiro de Pesquisas Fisicas, Rio de Janeiro, Brazil \\ ${ }^{11}$ Universidade do Estado do Rio de Janeiro, Rio de Janeiro, Brazil \\ ${ }^{12}$ Instituto de Fisica Teorica, Universidade Estadual Paulista, Sao Paulo, Brazil \\ ${ }^{13}$ Institute for Nuclear Research and Nuclear Energy, Sofia, Bulgaria \\ ${ }^{14}$ University of Sofia, Sofia, Bulgaria \\ ${ }^{15}$ Institute of High Energy Physics, Beijing, China \\ ${ }^{16}$ State Key Laboratory of Nuclear Physics and Technology,, Peking University, Beijing, China \\ ${ }^{17}$ Universidad de Los Andes, Bogota, Colombia \\ ${ }^{18}$ Technical University of Split, Split, Croatia \\ ${ }^{19}$ University of Split, Split, Croatia \\ ${ }^{20}$ Institute Rudjer Boskovic, Zagreb, Croatia \\ ${ }^{21}$ University of Cyprus, Nicosia, Cyprus \\ ${ }^{22}$ Charles University, Prague, Czech Republic \\ ${ }^{23}$ Academy of Scientific Research and Technology of the Arab Republic of Egypt, \\ Egyptian Network of High Energy Physics, Cairo, Egypt \\ ${ }^{24}$ National Institute of Chemical Physics and Biophysics, Tallinn, Estonia \\ ${ }^{25}$ Department of Physics, University of Helsinki, Helsinki, Finland \\ ${ }^{26}$ Helsinki Institute of Physics, Helsinki, Finland \\ ${ }^{27}$ Lappeenranta University of Technology, Lappeenranta, Finland \\ ${ }^{28}$ Laboratoire d'Annecy-le-Vieux de Physique des Particules, IN2P3-CNRS, Annecy-le-Vieux, France \\ ${ }^{29}$ DSM/IRFU, CEA/Saclay, Gif-sur-Yvette, France
}


${ }^{30}$ Laboratoire Leprince-Ringuet, Ecole Polytechnique, IN2P3-CNRS, Palaiseau, France

${ }^{31}$ Institut Pluridisciplinaire Hubert Curien, Université de Strasbourg, Université de Haute Alsace Mulhouse,

CNRS/IN2P3, Strasbourg, France

${ }^{32}$ Centre de Calcul de l'Institut National de Physique Nucleaire et de Physique des Particules (IN2P3), Villeurbanne, France

${ }^{33}$ Université de Lyon, Université Claude Bernard Lyon 1, CNRS-IN2P3, Institut de Physique Nucléaire de Lyon, Villeurbanne, France

${ }^{34}$ Institute of High Energy Physics and Informatization, Tbilisi State University, Tbilisi, Georgia

${ }^{35}$ RWTH Aachen University, I. Physikalisches Institut, Aachen, Germany

${ }^{36}$ RWTH Aachen University, III. Physikalisches Institut A, Aachen, Germany

${ }^{37}$ RWTH Aachen University, III. Physikalisches Institut B, Aachen, Germany

${ }^{38}$ Deutsches Elektronen-Synchrotron, Hamburg, Germany

${ }^{39}$ University of Hamburg, Hamburg, Germany

${ }^{40}$ Institut für Experimentelle Kernphysik, Karlsruhe, Germany

${ }^{41}$ Institute of Nuclear Physics "Demokritos," Aghia Paraskevi, Greece

${ }^{42}$ University of Athens, Athens, Greece

${ }^{43}$ University of Ioánnina, Ioánnina, Greece

${ }^{44}$ KFKI Research Institute for Particle and Nuclear Physics, Budapest, Hungary

${ }^{45}$ Institute of Nuclear Research ATOMKI, Debrecen, Hungary

${ }^{46}$ University of Debrecen, Debrecen, Hungary

${ }^{47}$ Panjab University, Chandigarh, India

${ }^{48}$ University of Delhi, Delhi, India

${ }^{49}$ Bhabha Atomic Research Centre, Mumbai, India

${ }^{50}$ Tata Institute of Fundamental Research-EHEP, Mumbai, India

${ }^{51}$ Tata Institute of Fundamental Research-HECR, Mumbai, India

${ }^{52}$ Institute for Research and Fundamental Sciences (IPM), Tehran, Iran

${ }^{53}$ INFN Sezione di Bari, Bari, Italy

${ }^{53 \mathrm{~b}}$ Università di Bari, Bari, Italy

${ }^{53 \mathrm{c}}$ Politecnico di Bari, Bari, Italy

${ }^{54 a}$ INFN Sezione di Bologna, Bologna, Italy

${ }^{54 \mathrm{~b}}$ Università di Bologna, Bologna, Italy

${ }^{55 a}$ INFN Sezione di Catania, Catania, Italy

${ }^{55 \mathrm{~b}}$ Università di Catania, Catania, Italy

${ }^{56 a}$ INFN Sezione di Firenze, Firenze, Italy

${ }^{56 \mathrm{~b}}$ Università di Firenze, Firenze, Italy

${ }^{57}$ INFN Laboratori Nazionali di Frascati, Frascati, Italy

${ }^{58}$ INFN Sezione di Genova, Genova, Italy

${ }^{59 a}$ INFN Sezione di Milano-Biccoca, Milano, Italy

${ }^{59 b}$ Università di Milano-Bicocca, Milano, Italy

${ }^{60 a}$ INFN Sezione di Napoli, Napoli, Italy

${ }^{60 \mathrm{~b}}$ Università di Napoli "Federico II," Napoli, Italy

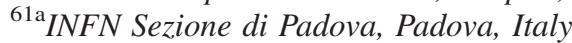

${ }^{61 \mathrm{~b}}$ Università di Padova, Padova, Italy

${ }^{61 \mathrm{c}}$ Università di Trento (Trento), Padova, Italy

${ }^{62 a}$ INFN Sezione di Pavia, Pavia, Italy

${ }^{62 \mathrm{~b}}$ Università di Pavia, Pavia, Italy

${ }^{63 a}$ INFN Sezione di Perugia, Perugia, Italy

${ }^{63 \mathrm{~b}}$ Università di Perugia, Perugia, Italy

${ }^{64 \mathrm{a}}$ INFN Sezione di Pisa, Pisa, Italy

${ }^{64 \mathrm{~b}}$ Università di Pisa, Pisa, Italy

${ }^{64 \mathrm{c}}$ Scuola Normale Superiore di Pisa, Pisa, Italy

${ }^{65 a}$ INFN Sezione di Roma, Roma, Italy

${ }^{65 b}$ Università di Roma "La Sapienza," Roma, Italy

${ }^{66 a}$ INFN Sezione di Torino, Torino, Italy

${ }^{66 \mathrm{~b}}$ Università di Torino, Torino, Italy

${ }^{66 c}$ Università del Piemonte Orientale (Novara), Torino, Italy

${ }^{67 \mathrm{a} I N F N}$ Sezione di Trieste, Trieste, Italy

${ }^{67 \mathrm{~b}}$ Università di Trieste, Trieste, Italy

${ }^{68}$ Kangwon National University, Chunchon, Korea

${ }^{69}$ Kyungpook National University, Daegu, Korea

${ }^{70}$ Chonnam National University, Institute for Universe and Elementary Particles, Kwangju, Korea

${ }^{71}$ Korea University, Seoul, Korea

${ }^{72}$ University of Seoul, Seoul, Korea 
${ }^{73}$ Sungkyunkwan University, Suwon, Korea

${ }^{74}$ Vilnius University, Vilnius, Lithuania

${ }^{75}$ Centro de Investigacion y de Estudios Avanzados del IPN, Mexico City, Mexico

${ }^{76}$ Universidad Iberoamericana, Mexico City, Mexico

${ }^{77}$ Benemerita Universidad Autonoma de Puebla, Puebla, Mexico

${ }^{78}$ Universidad Autónoma de San Luis Potosí, San Luis Potosí, Mexico

${ }^{79}$ University of Auckland, Auckland, New Zealand

${ }^{80}$ University of Canterbury, Christchurch, New Zealand

${ }^{81}$ National Centre for Physics, Quaid-I-Azam University, Islamabad, Pakistan

${ }^{82}$ Institute of Experimental Physics, Faculty of Physics, University of Warsaw, Warsaw, Poland

${ }^{83}$ Soltan Institute for Nuclear Studies, Warsaw, Poland

${ }^{84}$ Laboratório de Instrumentação e Física Experimental de Partículas, Lisboa, Portugal

${ }^{85}$ Joint Institute for Nuclear Research, Dubna, Russia

${ }^{86}$ Petersburg Nuclear Physics Institute, Gatchina (St. Petersburg), Russia

${ }^{87}$ Institute for Nuclear Research, Moscow, Russia

${ }^{88}$ Institute for Theoretical and Experimental Physics, Moscow, Russia

${ }^{89}$ Moscow State University, Moscow, Russia

${ }^{90}$ P.N. Lebedev Physical Institute, Moscow, Russia

${ }^{91}$ State Research Center of Russian Federation, Institute for High Energy Physics, Protvino, Russia

${ }^{92}$ University of Belgrade, Faculty of Physics and Vinca Institute of Nuclear Sciences, Belgrade, Serbia

${ }^{93}$ Centro de Investigaciones Energéticas Medioambientales y Tecnológicas (CIEMAT), Madrid, Spain

${ }^{94}$ Universidad Autónoma de Madrid, Madrid, Spain

${ }^{95}$ Universidad de Oviedo, Oviedo, Spain

${ }^{96}$ Instituto de Física de Cantabria (IFCA), CSIC-Universidad de Cantabria, Santander, Spain

${ }^{97}$ CERN, European Organization for Nuclear Research, Geneva, Switzerland

${ }^{98}$ Paul Scherrer Institut, Villigen, Switzerland

${ }^{99}$ Institute for Particle Physics, ETH Zurich, Zurich, Switzerland

${ }^{100}$ Universität Zürich, Zurich, Switzerland

${ }^{101}$ National Central University, Chung-Li, Taiwan

${ }^{102}$ National Taiwan University (NTU), Taipei, Taiwan

${ }^{103}$ Cukurova University, Adana, Turkey

${ }^{104}$ Middle East Technical University, Physics Department, Ankara, Turkey

${ }^{105}$ Bogazici University, Istanbul, Turkey

${ }^{106}$ National Scientific Center, Kharkov Institute of Physics and Technology, Kharkov, Ukraine

${ }^{107}$ University of Bristol, Bristol, United Kingdom

${ }^{108}$ Rutherford Appleton Laboratory, Didcot, United Kingdom

${ }^{109}$ Imperial College, London, United Kingdom

${ }^{110}$ Brunel University, Uxbridge, United Kingdom

${ }^{111}$ Baylor University, Waco, Texas 76706, USA

${ }^{112}$ Boston University, Boston, Massachusetts 02215, USA

${ }^{113}$ Brown University, Providence, Rhode Island 02912, USA

${ }^{114}$ University of California, Davis, Davis, California 95616, USA

${ }^{115}$ University of California, Los Angeles, Los Angeles, California 90095, USA

${ }^{116}$ University of California, Riverside, Riverside, California 92521, USA

${ }^{117}$ University of California, San Diego, La Jolla, California 92093, USA

${ }^{118}$ University of California, Santa Barbara, Santa Barbara, California 93106, USA

${ }^{119}$ California Institute of Technology, Pasadena, California 91125, USA

${ }^{120}$ Carnegie Mellon University, Pittsburgh, Pennsylvania 15213, USA

${ }^{121}$ University of Colorado at Boulder, Boulder, Colorado 80309, USA

${ }^{122}$ Cornell University, Ithaca, New York 14853-5001, USA

${ }^{123}$ Fairfield University, Fairfield, Connecticut 06824, USA

${ }^{124}$ Fermi National Accelerator Laboratory, Batavia, Illinois 60510-0500, USA

${ }^{125}$ University of Florida, Gainesville, Florida 32611-8440, USA

${ }^{126}$ Florida International University, Miami, Florida 33199, USA

${ }^{127}$ Florida State University, Tallahassee, Florida 32306-4350, USA

${ }^{128}$ Florida Institute of Technology, Melbourne, Florida 32901, USA

${ }^{129}$ University of Illinois at Chicago (UIC), Chicago, Illinois 60607-7059, USA

${ }^{130}$ The University of Iowa, Iowa City, Iowa 52242-1479, USA

${ }^{131}$ Johns Hopkins University, Baltimore, Maryland 21218, USA

${ }^{132}$ The University of Kansas, Lawrence, Kansas 66045, USA

${ }^{133}$ Kansas State University, Manhattan, Kansas 66506, USA 
${ }^{134}$ Lawrence Livermore National Laboratory, Livermore, California 94720, USA

${ }^{135}$ University of Maryland, College Park, Maryland 20742, USA

${ }^{136}$ Massachusetts Institute of Technology, Cambridge, Massachusetts 02139, USA

${ }^{137}$ University of Minnesota, Minneapolis, Minnesota 55455, USA

${ }^{138}$ University of Mississippi, University, Mississippi 38677, USA

${ }^{139}$ University of Nebraska-Lincoln, Lincoln, Nebraska 68588-0111, USA

${ }^{140}$ State University of New York at Buffalo, Buffalo, New York 14260-1500, USA

${ }^{141}$ Northeastern University, Boston, Massachusetts 02115, USA

${ }^{142}$ Northwestern University, Evanston, Illinois 60208-3112, USA

${ }^{143}$ University of Notre Dame, Notre Dame, Indiana 46556, USA

${ }^{144}$ The Ohio State University, Columbus, Ohio 43210, USA

${ }^{145}$ Princeton University, Princeton, New Jersey 08544-0708, USA

${ }^{146}$ University of Puerto Rico, Mayaguez, Puerto Rico 00680

${ }^{147}$ Purdue University, West Lafayette, Indiana 47907-1396, USA

${ }^{148}$ Purdue University Calumet, Hammond, Indiana 46323, USA

${ }^{149}$ Rice University, Houston, Texas 77251-1892, USA

${ }^{150}$ University of Rochester, Rochester, New York 14627-0171, USA

${ }^{151}$ The Rockefeller University, New York, New York 10021-6399, USA

${ }^{152}$ Rutgers, the State University of New Jersey, Piscataway, New Jersey 08854-8019, USA

${ }^{153}$ University of Tennessee, Knoxville, Tennessee 37996-1200, USA

${ }^{154}$ Texas A\&M University, College Station, Texas 77843-4242, USA

${ }^{155}$ Texas Tech University, Lubbock, Texas 79409-1051, USA

${ }^{156}$ Vanderbilt University, Nashville, Tennessee 37235, USA

${ }^{157}$ University of Virginia, Charlottesville, Virginia 22901, USA

${ }^{158}$ Wayne State University, Detroit, Michigan 48202, USA

${ }^{159}$ University of Wisconsin, Madison, Wisconsin 53706, USA

${ }^{\mathrm{a}}$ Deceased.

${ }^{\mathrm{b}}$ Also at CERN, European Organization for Nuclear Research, Geneva, Switzerland.

${ }^{\mathrm{c}}$ Also at Universidade Federal do ABC, Santo Andre, Brazil.

${ }^{\mathrm{d}}$ Also at Laboratoire Leprince-Ringuet, Ecole Polytechnique, IN2P3-CNRS, Palaiseau, France.

${ }^{\mathrm{e} A l s o}$ at Suez Canal University, Suez, Egypt.

${ }^{\mathrm{f}}$ Also at British University, Cairo, Egypt.

${ }^{\mathrm{g}}$ Also at Fayoum University, El-Fayoum, Egypt.

${ }^{\mathrm{h}}$ Also at Soltan Institute for Nuclear Studies, Warsaw, Poland.

${ }^{\mathrm{i}}$ Also at Massachusetts Institute of Technology, Cambridge, MA, USA.

${ }^{\mathrm{j}}$ Also at Université de Haute-Alsace, Mulhouse, France.

${ }^{\mathrm{k}}$ Also at Brandenburg University of Technology, Cottbus, Germany.

${ }^{1}$ Also at Moscow State University, Moscow, Russia.

${ }^{\mathrm{m}}$ Also at Institute of Nuclear Research ATOMKI, Debrecen, Hungary.

${ }^{\text {n} A l s o ~ a t ~ E o ̈ t v o ̈ s ~ L o r a ́ n d ~ U n i v e r s i t y, ~ B u d a p e s t, ~ H u n g a r y . ~}$

${ }^{\circ}$ Also at Tata Institute of Fundamental Research - HECR, Mumbai, India.

${ }^{\mathrm{p}}$ Also at University of Visva-Bharati, Santiniketan, India.

${ }^{\mathrm{q}}$ Also at Sharif University of Technology, Tehran, Iran.

${ }^{\mathrm{r}}$ Also at Shiraz University, Shiraz, Iran.

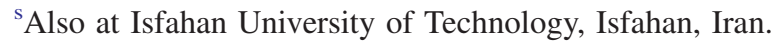

'Also at Facoltà Ingegneria Università di Roma "La Sapienza," Roma, Italy.

uAlso at Università della Basilicata, Potenza, Italy.

${ }^{\mathrm{v}}$ Also at Università degli studi di Siena, Siena, Italy.

${ }^{\mathrm{w}}$ Also at California Institute of Technology, Pasadena, CA, USA.

${ }^{\mathrm{x}}$ Also at Faculty of Physics of University of Belgrade, Belgrade, Serbia.

${ }^{\mathrm{y}}$ Also at University of California, Los Angeles, Los Angeles, CA, USA.

${ }^{\mathrm{z}}$ Also at University of Florida, Gainesville, FL, USA.

${ }^{\text {aa }}$ Also at Université de Genève, Geneva, Switzerland.

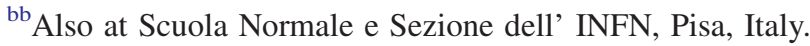

${ }^{\mathrm{cc}}$ Also at INFN Sezione di Roma, Università di Roma "La Sapienza", Roma, Italy.

${ }^{\mathrm{dd}}$ Also at University of Athens, Athens, Greece.

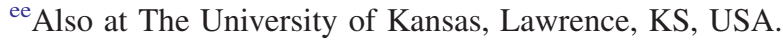


${ }^{\mathrm{ff}}$ Also at Institute for Theoretical and Experimental Physics, Moscow, Russia.

${ }^{\mathrm{gg}}$ Also at Paul Scherrer Institut, Villigen, Switzerland.

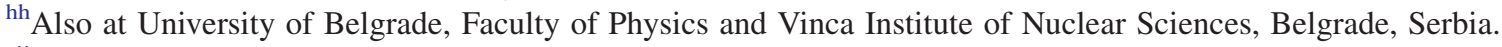

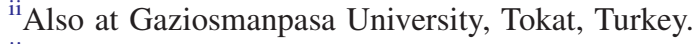

${ }^{\mathrm{jj}}$ Also at Adiyaman University, Adiyaman, Turkey.

${ }^{\mathrm{kk}}$ Also at Mersin University, Mersin, Turkey.

${ }^{11}$ Also at Izmir Institute of Technology, Izmir, Turkey.

${ }^{\mathrm{mm}}$ Also at Kafkas University, Kars, Turkey.

${ }^{\mathrm{nn}}$ Also at Suleyman Demirel University, Isparta, Turkey.

${ }^{\text {oo }}$ Also at Ege University, Izmir, Turkey.

${ }^{\mathrm{pp}}$ Also at Rutherford Appleton Laboratory, Didcot, United Kingdom.

${ }^{\mathrm{qq}}$ Also at School of Physics and Astronomy, University of Southampton, Southampton, United Kingdom.

${ }^{\mathrm{rr}}$ Also at INFN Sezione di Perugia, Università di Perugia, Perugia, Italy.

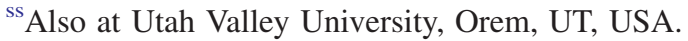

${ }^{t t}$ Also at Institute for Nuclear Research, Moscow, Russia.

${ }^{\mathrm{uu}}$ Also at Erzincan University, Erzincan, Turkey. 